\title{
Isogeometric locking-free plate element: a simple first order shear deformation theory for functionally graded plates
}

\author{
Shuohui Yin ${ }^{\mathrm{a}}$, Jack S. Hale ${ }^{\mathrm{b}}$, Tiantang Yu ${ }^{\mathrm{a},{ }^{*}}$, Tinh Quoc Bui ${ }^{\mathrm{c},}$, Stéphane P.A. Bordas ${ }^{\mathrm{b}, \mathrm{d}}$ \\ ${ }^{a}$ Department of Engineering Mechanics, Hohai University, Nanjing 210098, P.R. China. \\ ${ }^{\mathrm{b}}$ Faculté des Sciences, de la Technologie et de la Communication, University of Luxembourg, 6, rue Richard \\ Coudenhove-Kalergi, L-1359, Luxembourg. \\ ${ }^{\mathrm{c}}$ Department of Mechanical and Environmental Informatics, Graduate School of Information Science and \\ Engineering, Tokyo Institute of Technology, 2-12-1-W8-22, Ookayama, Meguro-ku, Tokyo 152-8552, Japan. \\ d Cardiff School of Engineering, Cardiff University, The Queen's Building, The Parade, Cardiff, Wales, \\ CF24 3AA, UK.
}

\begin{abstract}
An effective, simple, and locking-free plate formulation is proposed to analyze the static bending, buckling, and free vibration of homogeneous and functionally graded plates. The simple first-order shear deformation theory (S-FSDT), which was recently presented in Composite Structures (2013; 101:332-340), is naturally free from shear-locking and captures the physics of the shear-deformation effect present in the original FSDT, whilst also being less computationally expensive due to having fewer unknowns. The S-FSDT requires $\mathrm{C}^{1}$-continuity that is simple to satisfy with the inherent high-order continuity of the non-uniform rational B-spline (NURBS) basis functions, which we use in the framework of isogeometric analysis (IGA). Numerical examples are solved and the results are compared with reference solutions to confirm the accuracy of the proposed method. Furthermore, the effects of boundary conditions, gradient index, and geometric shape on the mechanical response of functionally graded plates are investigated.
\end{abstract}

Keywords: Functionally graded materials; First-order shear deformation theory; Isogeometric analysis; NURBS; Plates

\footnotetext{
*Corresponding authors. Tel.: +86 2583787901 (T.T. Yu); +81 357343587 (T.Q. Bui).

E-mail addresses: tiantangyu@ @hu.edu.cn (T.T. Yu), tinh.buiquoc@gmail.com (T.Q. Bui).
} 


\section{Introduction}

Functionally graded plates (FG plates) are a special type of composite structures with continuous variation of material properties between the top and bottom surfaces of the plate. Due to the advantageous mechanical behaviors of FG plates they are seeing increased use in a variety of engineering applications [1]. A significant number of studies have been performed to examine the mechanical behavior of FG plates see e.g., [2] for a review. It is widely accepted [2] that plate theories such as the first-order shear deformable theory (FSDT), sometimes also referred to as the Reissner-Mindlin theory, that take into account the shear-deformation effect are necessary to adequately capture the physical behavior of thick plates. Therefore the classical, or Kirchhoff plate theory, which does not capture the effect of shear deformations is not a suitable model for thick FG plates.

Historically the FSDT [3], sometimes also referred to as the Reissner-Mindlin theory, has been popular in computational mechanics for two main reasons: firstly, as mentioned above, it captures the extra physics of shear-deformation not present in the classical theory, and secondly, it relaxes the $\mathrm{C}^{1}$ continuity requirement of the classical theory to $\mathrm{C}^{0}$. This $\mathrm{C}^{0}$ continuity requirement is easier to satisfy using the low-order Lagrangian finite elements that form the basis of most finite element packages. However it is well-known that naïve numerical implementations of the standard FSDT using low-order Lagrangian shape functions typically suffer from shear-locking in the thin-plate or Kirchhoff limit resulting in totally incorrect solutions. Special techniques usually based on the application of a mixed variational formulation, such as the MITC family of elements [4], solve the shear-locking problem, but with additional expense and implementation complexity.

However, with the introduction of numerical methods relying on basis functions with natural $\mathrm{C}^{1}$ continuity such as NURBS in an isogeometric analysis framework (IGA) [5] and meshfree methods $[6,7]$ we believe that the physical accuracy and straightforward numerical implementation are no longer at odds. In this paper we develop a simple, efficient, and locking-free numerical method for thin through to thick shear-deformable plates by using a $\mathrm{C}^{1}$ continuity formulation that includes the effects of shear-deformation. We prove its efficacy by studying homogeneous and functionally graded plates.

The underlying differential equation in our formulation is based on the simple FSDT (S-FSDT) 
recently presented in $[8,9]$. The key idea in the derivation of the S-FSDT is the decomposition of

the transverse displacements in the FSDT into bending and shear parts before eliminating the rotation variables using the partial derivatives of the transverse bending displacement only. This weak formulation of the S-FSDT problem requires $C^{1}$ continuity just like in the classical plate theory but also includes the shear deformable physics of the FSDT. Therefore as well as being viewed as a simple FSDT, this formulation could also be viewed as a classical theory augmented with the shear-deformable physics of the FSDT formulation. Furthermore because the rotation variables of the standard FSDT are eliminated in terms of the bending transverse displacements the resulting weak formulation contains only four variables rather than the usual five, resulting in reduced computational expense.

In the thin-plate limit the S-FSDT recovers the classical plate theory just like the standard FSDT. However, because the thin-plate limit is included naturally in the S-FSDT formulation there is no need to resort to special numerical formulations to eliminate shear-locking as in the standard FSDT; as long as the basis functions satisfy $\mathrm{C}^{1}$ continuity the formulation will be free from shear-locking. Other authors have also used modified plate formulations to ease the construction of numerical methods; recently, Brezzi et al. [10] introduced the twist-Kirchhoff theory that uses a partial Kirchhoff hypothesis to create a simple thin-plate finite element method. Cho and Atluri [11] use a change of variables from transverse displacement to shear stress to develop a meshfree method for the Timoshenko beam problem that is free from shear-locking. This type of approach has been extended by Tiago and Leitão [12] to the plate problem.

Because of the requirement of $\mathrm{C}^{1}$ continuity we develop the S-FSDT within the framework of the isogeometric analysis (IGA) method proposed by Hughes et al. [5]. This method is becoming popular because of its many advantages, such as exact geometrical modeling, higher-order continuity, and simple mesh refinement. However, the primary reason for using the IGA method in this paper is to achieve the $C^{1}$ continuity condition required by the weak form of the S-FSDT. As such, other numerical methods with natural $\mathrm{C}^{1}$ continuity such as meshfree methods $[2,6]$ are also be excellent candidates for the discretization of the S-FSDT.

The principle of IGA involves the adoption of CAD basis functions such as non-uniform rational B-spline (NURBS) functions as the shape functions of finite element analysis. The IGA has been successfully implemented in many engineering problems including structural vibrations [13], plates and shells [14-19], fluid mechanics [20], fluid-structure interaction problems [21], damage and 
fracture mechanics[22], and structural shape optimization [23].

It is important to note that the usual IGA method also suffers from shear-locking when discretizing the standard FSDT problem, just like the standard Lagrangian finite element method. The most common remedy is to increase the polynomial order of consistency such that the basis functions are better able to represent the Kirchhoff limit. Echter and Bischoff [24] shows that this can result in sub-optimal convergence and reduce numerical efficiency in the IGA, and present a solution to the problem of shear-locking based on the Discrete Shear Gap (DSG) methodology for the 1D Timoshenko beam problem. Valizadeh et al. [14] use a modified shear correction factor dependent on the local discretization size to suppress shear locking. Beirão da Veiga et al. [25] present a method where the NURBS basis functions satisfy the Kirchhoff condition a priori. The resulting method is completely free of shear-locking, but requires a more complex basis function construction which involves a contravariant mapping for the basis functions interpolating the rotation variable. In contrast, the method we develop in this paper is considerably simpler and can be easily implemented using the existing functionality in open-source IGA frameworks such as igafem [26] and GeoPDEs [27].

In summary, the main objective of this study is to propose a new locking-free plate formulation for solving the static bending, buckling, and free vibration of both thin and thick FG plates. The new approach uses the high continuity of IGA to discretize the S-FSDT. The resulting S-FSDT-based IGA method has four degrees of freedom and is easy to implement within existing open-source IGA frameworks. We show the efficacy of the resulting method with extensive numerical examples focusing on functionally graded plates in static bending, free vibration, and buckling. We show the shear-locking free nature of the proposed method. The effects of boundary condition, gradient index, and geometric shape on the mechanical responses of FG plates are investigated numerically. The computed results are in typically within $1 \%$ of reference solutions in available in the literature.

The paper is structured as follows. Section 2 briefly presents the theoretical formulation. Section 3 describes NURBS-based isogeometric analysis in detail. Section 4 presents the validation of the locking-free characteristic of the proposed method. Section 5 shows the numerical results derived from the proposed IGA and in Section 6 we discuss the proposed method and suggest directions for future work. 


\section{Formulation}

\subsection{Functionally graded plate}

Consider a ceramic-metal FG plate with thickness $h$. The bottom and top faces of the plate are assumed to be fully metallic and ceramic, respectively. The $x y$-plane is the mid-plane of the plate, and the positive $z$-axis is upward from the mid-plane. In this study, Poisson's ratio $v$ is constant and Young's modulus $E$ and density $\rho$ vary through the thickness with a power law distribution:

$$
\begin{aligned}
& E(z)=E_{m}+\left(E_{c}-E_{m}\right)\left(\frac{1}{2}+\frac{z}{h}\right)^{n}, \\
& \rho(z)=\rho_{m}+\left(\rho_{c}-\rho_{m}\right)\left(\frac{1}{2}+\frac{z}{h}\right)^{n},
\end{aligned}
$$

where $n$ is the gradient index, $z$ is the thickness coordinate variable with $-h / 2 \leq z \leq h / 2$, and subscripts $c$ and $m$ represent the ceramic and metal constituents, respectively.

\subsection{Brief on the S-FSDT}

In this subsection, we briefly present the theoretical formula of the S-FSDT. For more details, we refer the reader to $[8,9]$. In the standard FSDT the three-dimensional displacement field $(u, v, w)$ can be expressed in terms of five unknown variables as follows:

$$
\begin{aligned}
& u(x, y, z)=u_{0}(x, y)+z \phi_{x}(x, y) \\
& v(x, y, z)=v_{0}(x, y)+z \phi_{y}(x, y), \\
& w(x, y, z)=w_{0}(x, y)
\end{aligned}
$$

where $u_{0}, v_{0}$, and $w_{0}$ represent the displacements at the mid-plane of a plate in the $x, y$, and $z$ directions respectively; $\phi_{x}$ and $\phi_{y}$ denote the transverse normal rotations of the $y$ and $x$ axes.

To derive the simple FSDT (S-FSDT), the following assumptions are made to simplify the FSDT: (a) the transverse displacement $w_{0}$ is divided into bending component $w_{b}$ and shear component $w_{s}$, i.e., $w_{0}=w_{b}+w_{s}$; (b) The rotation variable in the FSDT is expressed in terms of the bending component only $\phi_{x}=-\partial w_{b} / \partial x, \phi_{y}=-\partial w_{b} / \partial y$. Therefore, Eq. (3) can be rewritten as follows: 


$$
\begin{aligned}
& u(x, y, z)=u_{0}(x, y)-z \partial w_{b}(x, y) / \partial x \\
& v(x, y, z)=v_{0}(x, y)-z \partial w_{b}(x, y) / \partial y . \\
& w(x, y, z)=w_{b}(x, y)+w_{s}(x, y)
\end{aligned}
$$

In contrast with the FSDT, the displacement fields in Eq. (4) for the S-FSDT contain only four unknowns, namely, $u_{0}, v_{0}, w_{b}, w_{s}$. Because the rotations are obtained by using the partial derivatives of the bending component $w_{b}$ conforming discretizations of the S-FSDT are inherently free from the issue of shear-locking.

The three physically relevant boundary conditions for the S-FSDT are the clamped, simply supported and free conditions, as in the Kirchhoff-Love or classical plate theory. In contrast, the FSDT has five physically relevant boundary conditions, hard clamped, soft clamped, hard simply supported, soft simply supported and free. Interested readers are referred to Arnold and Falk [28] for an in-depth discussion. Although the boundary conditions in the FSDT are more descriptive than the S-FSDT, in practical terms we have not found this to be an issue as demonstrated in the results section where good agreement with the FSDT is achieved.

By making the usual small strain assumptions, the strain-displacement relations are expressed as follows:

$$
\left\{\begin{array}{l}
\varepsilon_{x} \\
\varepsilon_{y} \\
\varepsilon_{x y} \\
\gamma_{x z} \\
\gamma_{y z}
\end{array}\right\}=\left\{\begin{array}{c}
\frac{\partial u_{0}}{\partial x}-z \frac{\partial^{2} w_{b}}{\partial x^{2}} \\
\frac{\partial v_{0}}{\partial y}-z \frac{\partial^{2} w_{b}}{\partial y^{2}} \\
\frac{\partial u_{0}}{\partial y}+\frac{\partial v_{0}}{\partial x}-2 z \frac{\partial^{2} w_{b}}{\partial x \partial y} \\
\frac{\partial w_{s}}{\partial x} \\
\frac{\partial w_{s}}{\partial y}
\end{array}\right\}
$$

Then Eq. (5) can be written in the following matrix form:

$$
\boldsymbol{\varepsilon}=\left\{\begin{array}{c}
\boldsymbol{\varepsilon}_{0} \\
\mathbf{0}
\end{array}\right\}+\left\{\begin{array}{c}
-z \mathbf{\kappa} \\
\gamma
\end{array}\right\},
$$

with 


$$
\boldsymbol{\varepsilon}_{0}=\left\{\begin{array}{c}
\frac{\partial u_{0}}{\partial x} \\
\frac{\partial v_{0}}{\partial y} \\
\frac{\partial u_{0}}{\partial y}+\frac{\partial v_{0}}{\partial x}
\end{array}\right\} ; \quad \boldsymbol{\kappa}=\left\{\begin{array}{c}
\frac{\partial^{2} w_{b}}{\partial x^{2}} \\
\frac{\partial^{2} w_{b}}{\partial y^{2}} \\
2 \frac{\partial^{2} w_{b}}{\partial x \partial y}
\end{array}\right\} ; \quad \gamma=\left\{\begin{array}{c}
\frac{\partial w_{s}}{\partial x} \\
\frac{\partial w_{s}}{\partial y}
\end{array}\right\} .
$$

The constitutive relations are derived from Hooke's law by the following equation:

$$
\boldsymbol{\sigma}=\mathbf{D}_{m}(z)\left(\boldsymbol{\varepsilon}_{0}-z \mathbf{\kappa}\right), \boldsymbol{\tau}=\mathbf{D}_{s}(z) \boldsymbol{\gamma},
$$

with

$$
\begin{aligned}
& \boldsymbol{\sigma}=\left[\begin{array}{lll}
\sigma_{x} & \sigma_{y} & \sigma_{x y}
\end{array}\right]^{T}, \boldsymbol{\tau}=\left[\begin{array}{ll}
\tau_{x z} & \tau_{y z}
\end{array}\right]^{T} \\
& \mathbf{D}_{m}(z)=\frac{E(z)}{1-v^{2}}\left[\begin{array}{ccc}
1 & v & 0 \\
v & 1 & 0 \\
0 & 0 & (1-v) / 2
\end{array}\right], \\
& \mathbf{D}_{s}(z)=\frac{k E(z)}{2(1+v)}\left[\begin{array}{ll}
1 & 0 \\
0 & 1
\end{array}\right]
\end{aligned}
$$

where $k$ is the shear correction factor. In this paper, we set $k=5 / 6$.

\section{NURBS-based isogeometric analysis}

In this section we give an overview of the NURBS basis function construction and derive the discrete weak form for the S-FSDT numerical formulation.

\subsection{NURBS basis function}

In $1 \mathrm{D}$ parametric space $\xi \in[0,1]$, a knot vector $\boldsymbol{k}(\xi)$ is a set of non-decreasing numbers that are between zero and one:

$$
\boldsymbol{k}(\xi)=\left\{\xi_{1}=0, \ldots, \xi_{i}, \ldots, \xi_{n+p+1}=1\right\}^{T},
$$

where $i$ is the knot index, $\xi_{i}$ is the $i^{\text {th }}$ knot, $n$ is the number of basis functions, and $p$ is the order of the polynomial.

The knot vector $\boldsymbol{k}(\xi)$ is called an open knot vector when the two ends of the knot are repeated $p+1$ times. Basis functions that are defined with an open knot vector are interpolatory at the beginning and end of the parametric space interval; thus, the open knot vector is used here. 
By using the given knot vector $\boldsymbol{k}(\xi)$, the $i^{\text {th }}$ B-spline basis function of degree $p$, written as $N_{i, p}(\xi)$, is defined recursively as follows [29]:

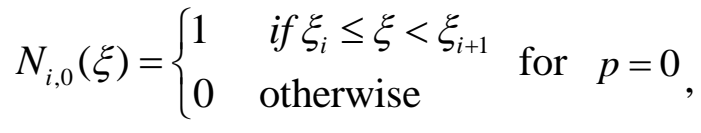

and

$$
N_{i, p}(\xi)=\frac{\xi-\xi_{i}}{\xi_{i+p}-\xi_{i}} N_{i, p-1}(\xi)+\frac{\xi_{i+p+1}-\xi}{\xi_{i+p+1}-\xi_{i+1}} N_{i+1, p-1}(\xi) \text { for } p \geq 1
$$

The NURBS basis function $R_{i, p}(\xi)$ in the framework of partition of unity is constructed by a weighted average of the B-spline basis functions [29]:

$$
R_{i, p}(\xi)=\frac{N_{i, p}(\xi) w_{i}}{\sum_{j=1}^{n} N_{j, p}(\xi) w_{j}},
$$

where $w_{i}$ is the $i^{\text {th }}$ weight, and $0<w_{i} \leq 1$.

Similarly, 2D NURBS basis functions can be constructed by taking the tensor product of two 1D B-spline basis functions as follows:

$$
R_{i, j}^{p, q}(\xi, \eta)=\frac{N_{i, p}(\xi) N_{j, q}(\eta) w_{i, j}}{\sum_{i=1}^{n} \sum_{j=1}^{m} N_{i, p}(\xi) N_{j, q}(\eta) w_{i, j}},
$$

where $w_{i, j}$ represents the 2D weight; $N_{i, p}(\xi)$ and $N_{j, q}(\eta)$ are the B-spline basis functions of order $p$ in the $\xi$ direction and order $q$ in the $\eta$ direction, respectively; $N_{j, q}(\eta)$ follows the recursive formula shown in Eqs. (11) and (12) with knot vector $\boldsymbol{k}(\eta)$. The definition of $\boldsymbol{k}(\eta)$ is similar to that of $k(\xi)$.

By using the NURBS basis functions, a NURBS surface of order $p$ in the $\xi$ direction and order $q$ in the $\eta$ direction can be constructed as follows:

$$
\boldsymbol{S}(\xi, \eta)=\sum_{i=1}^{n} \sum_{j=1}^{m} R_{i, j}^{p, q}(\xi, \eta) \boldsymbol{B}_{i, j}
$$

where $\boldsymbol{B}_{i, j}$ represents the coordinates of control points in two dimensions. 


\subsection{Discrete equations}

The parametric domain in IGA is similar to the isoparametric space in the finite element method (FEM). Thus, the generalized displacements in the middle plane are approximated as follows:

$$
\mathbf{u}_{0}^{h}=\sum_{I=1}^{N P} R_{I} \mathbf{u}_{I},
$$

with

$$
\begin{aligned}
\mathbf{u}_{0}^{h} & =\left[\begin{array}{llll}
u_{0}^{h} & v_{0}^{h} & w_{b}^{h} & w_{s}^{h}
\end{array}\right]^{T}, \\
\mathbf{u}_{I} & =\left[\begin{array}{llll}
u_{I} & v_{I} & w_{b I} & w_{s I}
\end{array}\right]^{T} .
\end{aligned}
$$

where $N P=(p+1)(q+1)$ is the number of control points per element, and $R_{I}$ and $\mathbf{u}_{I}$ denote the shape function and the unknown displacement vector at control point $I$, respectively.

By substituting Eq. (16) into Eq. (7), one can obtain the following:

$$
\boldsymbol{\varepsilon}_{0}=\sum_{I=1}^{N P} \mathbf{B}_{I}^{m} \mathbf{u}_{I}, \quad \boldsymbol{\kappa}=\sum_{I=1}^{N P} \mathbf{B}_{I}^{b} \mathbf{u}_{I}, \gamma=\sum_{I=1}^{N P} \mathbf{B}_{I}^{s} \mathbf{u}_{I}
$$

with

$$
\mathbf{B}_{I}^{m}=\left[\begin{array}{cccc}
R_{I, x} & 0 & 0 & 0 \\
0 & R_{I, y} & 0 & 0 \\
R_{I, y} & R_{I, x} & 0 & 0
\end{array}\right], \mathbf{B}_{I}^{b}=\left[\begin{array}{cccc}
0 & 0 & R_{I, x x} & 0 \\
0 & 0 & R_{I, y y} & 0 \\
0 & 0 & 2 R_{I, x y} & 0
\end{array}\right], \mathbf{B}_{I}^{s}=\left[\begin{array}{cccc}
0 & 0 & 0 & R_{I, x} \\
0 & 0 & 0 & R_{I, y}
\end{array}\right],
$$

For the static problem, the weak form can be expressed as follows:

$$
\int_{\Omega} \delta \boldsymbol{\varepsilon}^{T} \mathbf{D} \boldsymbol{\varepsilon} d \Omega+\int_{\Omega} \delta \boldsymbol{\gamma}^{T} \mathbf{D}^{s} \gamma d \Omega=\int_{\Omega} \delta\left(w_{b}+w_{s}\right) f d \Omega,
$$

where $f$ is the transverse loading per unit area and

$$
\boldsymbol{\varepsilon}=\left[\begin{array}{l}
\boldsymbol{\varepsilon}_{0} \\
\mathbf{\kappa}
\end{array}\right], \mathbf{D}=\left[\begin{array}{cc}
\mathbf{D}^{m} & \overline{\mathbf{B}} \\
\overline{\mathbf{B}} & \mathbf{D}^{b}
\end{array}\right], \mathbf{D}^{s}=\int_{-h / 2}^{h / 2} \mathbf{D}_{s}(z) d z,
$$

with

$$
\mathbf{D}^{m}=\int_{-h / 2}^{h / 2} \mathbf{D}_{m}(z) d z, \overline{\mathbf{B}}=\int_{-h / 2}^{h / 2} z \mathbf{D}_{m}(z) d z, \quad \mathbf{D}^{b}=\int_{-h / 2}^{h / 2} z^{2} \mathbf{D}_{m}(z) d z .
$$

For the free vibration analysis, a weak form can be expressed as follows:

$$
\int_{\Omega} \delta \boldsymbol{\varepsilon}^{T} \mathbf{D} \boldsymbol{\varepsilon} d \Omega+\int_{\Omega} \delta \boldsymbol{\varepsilon}^{T} \mathbf{D}^{s} \boldsymbol{\varepsilon} d \Omega=\int_{\Omega} \delta \mathbf{u}^{T} \mathbf{m} \ddot{\mathbf{u}} d \Omega,
$$

where

$$
\mathbf{m}=\left[\begin{array}{ll}
I_{0} & I_{1} \\
I_{1} & I_{2}
\end{array}\right], \quad\left(I_{0}, I_{1}, I_{2}\right)=\int_{-h / 2}^{h / 2} \rho(z)\left(1, z, z^{2}\right) d z
$$




$$
\mathbf{u}=\left[\begin{array}{l}
\mathbf{u}_{1} \\
\mathbf{u}_{2}
\end{array}\right], \mathbf{u}_{1}=\left\{\begin{array}{c}
u_{0}^{h} \\
v_{0}^{h} \\
w_{b}^{h}+w_{s}^{h}
\end{array}\right\}=\sum_{I=1}^{N P} \mathbf{N}_{I}^{1} \mathbf{u}_{I}, \mathbf{u}_{2}=\left\{\begin{array}{c}
\partial w_{b}^{h} / \partial x \\
\partial w_{b}^{h} / \partial y \\
0
\end{array}\right\}=\sum_{I=1}^{N P} \mathbf{N}_{I}^{2} \mathbf{u}_{I},
$$

with

$$
\mathbf{N}_{I}^{1}=\left[\begin{array}{cccc}
R_{I} & 0 & 0 & 0 \\
0 & R_{I} & 0 & 0 \\
0 & 0 & R_{I} & R_{I}
\end{array}\right], \quad \mathbf{N}_{I}^{2}=\left[\begin{array}{cccc}
0 & 0 & R_{I, x} & 0 \\
0 & 0 & R_{I, y} & 0 \\
0 & 0 & 0 & 0
\end{array}\right]
$$

Finally, for the buckling analysis, the weak form can be expressed as follows:

$$
\int_{\Omega} \delta \boldsymbol{\varepsilon}^{T} \mathbf{D} \boldsymbol{\varepsilon} d \Omega+\int_{\Omega} \delta \boldsymbol{\gamma}^{T} \mathbf{D}^{s} \gamma d \Omega+\int_{\Omega} \nabla^{T} \delta\left(w_{b}+w_{s}\right) \hat{\boldsymbol{\sigma}}_{0} \nabla\left(w_{b}+w_{s}\right) d \Omega=0 .
$$

where $\nabla^{T}=\left[\begin{array}{ll}\partial / \partial x & \partial / \partial y\end{array}\right]^{T}$ is the gradient operator and $\hat{\boldsymbol{\sigma}}_{0}=\left[\begin{array}{cc}\sigma_{x}^{0} & \tau_{x y}^{0} \\ \tau_{x y}^{0} & \sigma_{y}^{0}\end{array}\right]$ is the pre-buckling stresses under the in-plane.

By substituting Eqs. (18) and (21) into Eqs. (20), (23), and (27), the formulations of the static, free vibration, and buckling problems are rewritten in the following form:

$$
\begin{aligned}
& \mathbf{K d}=\mathbf{F}, \\
& \left(\mathbf{K}-\omega^{2} \mathbf{M}\right) \mathbf{d}=0, \\
& \left(\mathbf{K}-\lambda_{c r} \mathbf{K}_{g}\right) \mathbf{d}=0,
\end{aligned}
$$

where the global stiffness matrix $\mathbf{K}$ is given by the following:

$$
\mathbf{K}=\int_{\Omega}\left\{\begin{array}{l}
\mathbf{B}^{m} \\
\mathbf{B}^{b}
\end{array}\right\}^{T}\left[\begin{array}{cc}
\mathbf{D}^{m} & \overline{\mathbf{B}} \\
\overline{\mathbf{B}} & \mathbf{D}^{b}
\end{array}\right]\left\{\begin{array}{l}
\mathbf{B}^{m} \\
\mathbf{B}^{b}
\end{array}\right\} d \Omega+\int_{\Omega}\left(\mathbf{B}^{s}\right)^{T} \mathbf{D}^{s} \mathbf{B}^{s} d \Omega
$$

The load vector $\mathbf{F}$ is computed as follows:

$$
\mathbf{F}=\int_{\Omega} f \mathbf{N} d \Omega \text {, and } \mathbf{N}_{I}=\left[\begin{array}{llll}
0 & 0 & R_{I} & R_{I}
\end{array}\right]^{T} .
$$

The global mass matrix $\mathbf{M}$ is expressed as follows:

$$
\mathbf{M}=\int_{\Omega}\left\{\begin{array}{l}
\mathbf{N}^{1} \\
\mathbf{N}^{2}
\end{array}\right\}^{T} \mathbf{m}\left\{\begin{array}{l}
\mathbf{N}^{1} \\
\mathbf{N}^{2}
\end{array}\right\} d \Omega
$$

The geometric stiffness matrix $\mathbf{K}_{g}$ is expressed as follows:

$$
\mathbf{K}_{g}=\int_{\Omega}\left(\mathbf{B}^{g}\right)^{T} \hat{\boldsymbol{\sigma}}_{0} \mathbf{B}^{g} d \Omega
$$

with 


$$
\mathbf{B}_{I}^{g}=\left[\begin{array}{llll}
0 & 0 & R_{I, x} & R_{I, x} \\
0 & 0 & R_{I, y} & R_{I, y}
\end{array}\right] .
$$

\section{Validation of the fully locking-free property}

A homogeneous square plate with length $a$ and thickness $h$ under a uniform load $P=1 \mathrm{~N}$ is considered to test the locking-free characteristic of the developed approach. The material parameters used for this particular study are Young's modulus $E=1.092 \times 10^{6} \mathrm{~N} / \mathrm{mm}^{2}$ and Poisson's ratio $v=0.3$. The simply supported and clamped boundary conditions are considered. The rotations are obtained by using the derivatives of bending deflection $\phi_{x}=-\partial w_{b} / \partial x$ and $\phi_{y}=-\partial w_{b} / \partial y$; thus, the constraint on the rotations in the clamped boundary condition is imposed by fixing the z-component of the second row of control points as in [17]. The central deflection is normalized by $\bar{w}=\frac{100 w_{c} E h^{3}}{12\left(1-v^{2}\right) P a^{4}}$.

Quadratic and cubic NURBS basis functions are used in this study. The control points and physical mesh for the cubic NURBS basis function are shown in Fig. 1. The computed results of the normalized central deflections obtained using the IGA based on both the S-FSDT and original FSDT are compared with the analytical solutions [30] (Table 1). Some interesting issues may be observed from the results:

1. the FSDT-based IGA suffers shear locking when the length-thickness ratio $a / h$ is greater than 100 for the quadratic NURBS basis functions and $a / h$ is greater than 1000 for the cubic NURBS basis functions. We conclude that increasing the polynomial order is not sufficient to completely eliminate shear-locking when using the FSDT-based IGA.

2. the S-FSDT-based IGA is completely free from shear-locking when using both quadratic and cubic NURBS basis functions; therefore our S-FSDT-based IGA based method guarantees shear-locking free results, unlike the FSDT-based IGA method.

3. Increasing the polynomial order of the basis functions increases the accuracy of the proposed S-FSDT IGA method.

We also verify that the S-FSDT converges to the values given by the FSDT for thick plates and Kirchhoff-Love theory for thin plates. The normalized central deflections, bending deflections 
$\bar{w}_{b}=\frac{100 w_{b} E h^{3}}{12\left(1-v^{2}\right) P a^{4}}$, and shear deflections $\bar{w}_{s}=\frac{100 w_{s} E h^{3}}{12\left(1-v^{2}\right) P a^{4}}$ obtained by the IGA based on the S-FSDT with different length-thickness ratios a/h are show in Table 2. Table 2 shows the following: (1) when $a / h<20$, the shear deformation effects are considered in S-FSDT, similar to FSDT; (2) the effects of the shear component decreases with increasing $a / h$; (3) when $a / h>20$, the effects of shear component are negligible, and the S-FSDT converges to the value given by the Kirchhoff-Love theory.

\section{Numerical applications}

In this section, the static bending, free vibration, and buckling behavior of homogeneous and FG plates with different geometric shapes are examined by using the developed "S-FSDT-based IGA" model with cubic NURBS basis function. A $4 \times 4$ Gaussian quadrature scheme is used in each NURBS element to integrate the weak form. An original "FSDT-based IGA" computer code is implemented to the same problems for comparison purposes. In the following examples, the boundaries of the plate are denoted as simply supported (S), clamped (C), and free (F). A mesh of $16 \times 16$ control points is used for the square plates unless stated otherwise.

\subsection{Static analysis}

\subsubsection{Convergence and accuracy study}

To validate the convergence of the S-FSDT-based IGA, a fully simply supported $\mathrm{Al} / \mathrm{Al}_{2} \mathrm{O}_{3}$ square plate with a length-thickness ratio of $a / h=100$ and different gradient indexes $n$ under sinusoidal loads $P \sin (\pi x) \sin (\pi y)$ is considered. The material properties of Al are $v_{m}=0.3, E_{m}=70 G P a$, and $\rho_{m}=2707 \mathrm{~kg} / \mathrm{m}^{3}$, and the material properties of $\mathrm{Al}_{2} \mathrm{O}_{3}$ are $v_{c}=0.3, E_{c}=380 G P a$, $\rho_{c}=3800 \mathrm{~kg} / \mathrm{m}^{3}$. The central deflection is normalized by $\bar{w}=10 w_{c} E_{c} h^{3} /\left(P a^{4}\right)$. Table 3 presents the comparison of the normalized central deflections derived from the S-FSDT-based IGA, the FSDT-based IGA, and the analytical solutions [8, 31, 32]. The numerical results in the convergence study of the relative error of normalized central deflection $\left|\frac{\bar{w}-\bar{w}_{\text {exact }}}{\bar{w}_{\text {exact }}}\right|$ are shown in Fig. 2, and the analytical solutions based on S-FSDT [8] are adopted. Table 3 shows that the results obtained with 
S-FSDT are similar to the analytical solutions and match well with the results derived from the

FSDT-based IGA. The amplitude of the normalized central deflections increases with increasing values of the gradient index.

Another example is performed to illustrate the accuracy of the developed approach. In this example, the conditions are the same as the previous example except for the length-thickness ratio $a / h$. Table 4 shows the normalized central deflections obtained with the S-FSDT-based IGA, the FSDT-based IGA, and the analytical solutions $[8,31,32]$. The results from the present method are in good agreement with the analytical solutions [8, 31, 32] for both thin and thick plates, thus confirming the accuracy of the proposed approach.

\subsubsection{Bending analysis}

In this subsection the bending behavior of square and circular FG plates is investigated.

\subsubsection{Square plate}

An $\mathrm{Al} / \mathrm{ZrO}_{2}-1$ square plate with different boundary conditions, length-thickness ratios $a / h$, and gradient indexes $n$ under a uniform load is analyzed. The material properties of $\mathrm{Al}$ are $v_{m}=0.3$ and $E_{m}=70 G P a$ and those of $\mathrm{ZrO}_{2}-1$ are $v_{c}=0.3$ and $E_{c}=200 G P a$. The central deflection is normalized by $\bar{w}=\frac{100 w_{c} E_{m} h^{3}}{12\left(1-v_{m}^{2}\right) P a^{4}}$. Table 5 shows the normalized central deflections obtained with the S-FSDT-based IGA, FSDT-based IGA, FSDT-based kp-Ritz method [33], and FSDT-based edge-based smoothed FEM [34]. The results obtained with the S-FSDT-based IGA match well with those derived from other techniques. For all boundary conditions and length-thickness ratios, remarkable agreements are achieved between the S-FSDT-based IGA and FSDT-based IGA techniques. When the boundary condition changes from SSSS to SFSS and SFSF, the structural stiffness is gradually reduced; thus, the deflection magnitude is gradually increased. The deflection magnitude is increased by increasing the gradient index $n$.

To further examine the effect of the boundary conditions and gradient index on the central deflection of the FG plate, an $\mathrm{Al} / \mathrm{Al}_{2} \mathrm{O}_{3}$ thin plate with a length-thickness ratio of $a / h=100$ under a uniform load $P$ is considered. The central deflection is normalized by $\bar{w}=\frac{10 w_{c} E_{c} h^{3}}{P a^{4}}$. The normalized central deflections obtained by the S-FSDT-based IGA and FSDT-based IGA are 
presented in Table 6. The S-FSDT and FSDT deliver a similar result for all boundary conditions and gradient indices. A similar phenomenon to that of the $\mathrm{Al} / \mathrm{ZrO}_{2}-1$ plate is observed, i.e., when the boundary condition changes from CCCC and SCSC to SSSS and SFSF, the magnitude of the deflection is gradually increased. Fig. 3 shows the shapes of transverse displacement for the various boundary conditions and gradient index $n=2$.

\subsubsection{Circular plate}

To demonstrate the applicability of the present method to model common engineering shapes, the bending behavior of circular plate is studied in this subsection.

First, a homogeneous circular plate with thickness $h=0.01 \mathrm{~m}$ and radius $R=1 \mathrm{~m}$ subjected to a uniform transverse pressure $P=100 \mathrm{~N}$ is considered. Two different boundary conditions are considered: fully simply supported and fully clamped on the entire edges. The material properties of the plate are $E=1.092 \times 10^{6} \mathrm{~N} / \mathrm{m}^{2}$ and $v=0.3$. Fig. 4 shows the mesh of 121 control points. The central deflection is normalized by $\bar{w}=\frac{w_{c} E h^{3}}{P R^{4} 12\left(1-v^{2}\right)}$. The comparisons between the normalized central deflections obtained with the S-FSDT-based IGA, FSDT-based IGA, analytical solutions [35], and Kirchhoff theory-based MLPG [35] are listed in Table 7. The obtained results from the developed method are in good agreement with the analytical and MLPG solutions.

We then investigate the bending behavior of titanium/zirconium circular plate with different $h / R$ under roller supported and clamped boundary conditions subjected to a uniform pressure on the top surface of the plate. The material properties of titanium are $v_{m}=0.288$ and $E_{m}=110.25 \mathrm{GPa}$ and those of zirconium are $v_{c}=0.288$ and $E_{c}=278.41 G P a$. The deflection shapes of the plates are plotted in Fig. 5. Table 8 shows the normalized deflection $\bar{w}=\frac{64 w_{c} E h^{3}}{12\left(1-v^{2}\right) P R^{4}}$ at the center of the plate. A good agreement with HSDT based IGA [15], elasticity solutions [36] and semi-analytical numerical method [37] is obtained. A similar deflection pattern to that of the square plate is observed.

\subsection{Free vibration}

\subsubsection{Square plate}


A fully simply supported $\mathrm{Al} / \mathrm{Al}_{2} \mathrm{O}_{3}$ square plate with different length-thickness ratios $a / h$ is

analyzed. The obtained results of the first normalized natural frequencies $\omega^{*}=\omega h \sqrt{\rho_{c} / E_{c}}$ with meshes of $6,8,12,16$, and 24 control points per side are listed in Table 9, which shows fast the convergence of the results. The first normalized natural frequencies $\omega^{*}=\omega h \sqrt{\rho_{c} / E_{c}}$ obtained with different methods for several specified gradient indices are listed in Table 10. A good agreement among the obtained results with other reference solutions is evident, e.g., the magnitude of natural frequency decreases with increasing length-thickness ratios $a / h$. The reference solutions are taken from the analytical methods with high order and third shear deformation theories [38, 39], FEM with the S-FSDT [40], and element-free kp-Ritz method with FSDT [41] (Table 10). The model analysis shows that the amplitude of the first normalized natural frequency increases with the increasing value of the gradient index, similar to static bending.

To investigate the effects of the boundary conditions on the natural frequency, an $\mathrm{Al} / \mathrm{Al}_{2} \mathrm{O}_{3}$ square thin plate with a length-thickness ratio of $a / h=100$ under different boundary conditions and gradient indexes is considered. The first five mode normalized natural frequencies $\omega^{*}=\omega \pi^{2}\left(a^{2} / h\right) \sqrt{\rho_{m} / E_{m}}$ obtained with different methods are listed in Table 11. The results obtained with the S-FSDT-based IGA are in good agreement with the results from the FSDT-based IGA, CPT-neu-based IGA [42], and analytical solutions [43]. When the boundary conditions change from CCCC to SCSC, SSSS, and SFSF, the magnitude of the natural frequency gradually decreases. The magnitude of the natural frequency decreases with an increasing gradient index.

The first six mode shapes of fully simply supported and fully clamped square $\mathrm{Al} / \mathrm{Al}_{2} \mathrm{O}_{3}$ thin plates with a length-thickness ratio of $a / h=100$ and a gradient index of $n=2$ are given in Figs. 6 and 7 , respectively.

\subsubsection{Circular plate}

We consider a clamped $\mathrm{Al} / \mathrm{Al}_{2} \mathrm{O}_{3}$ circular plate with different thickness-radius ratios $h / R$. The frequency is normalized by $\omega^{*}=100 \omega h \sqrt{\rho_{c} / E_{c}}$. The obtained results shown in Table 12 agree with the results obtained by the FSDT-based IGA, HSDT-based IGA [15], FSDT-based semi-analytical solutions [44], FEM with ABAQUS, and uncoupled model (UM) with FSDT proposed by Ebrahimi et al.[45]. Decreasing the length-thickness ratio $\mathrm{h} / \mathrm{R}$ increases the magnitude 
of the natural frequency. The first six mode shapes of the circular plate are plotted in Fig. 8.

The first normalized frequencies $\omega^{*}=100 \omega h \sqrt{\rho_{c} / E_{c}}$ of the $\mathrm{Al}_{2} \mathrm{Al}_{2} \mathrm{O}_{3}$ circular plate with different boundary conditions and gradient indexes $n$ are tested and shown in Table 13. The magnitude of natural frequency decreases with an increasing gradient index. The natural frequencies of free and simply supported plates are lower than that of the clamped boundary plate.

\subsubsection{Square plate with a complicated cutout}

To illustrate the applicability of the proposed method for realistic geometries, a thin homogenous square plate with a complicated cutout is considered (Fig. 9). The material and geometrical parameters are Young's modulus $E=200 \times 10^{9} \mathrm{~Pa}$, Poisson's ratio $v=0.3$, mass density $\rho=$ $8,000 \mathrm{~kg} / \mathrm{m}^{3}$, and thickness $h=0.05 \mathrm{~m}$. The plate consists of 8 NURBS patches (as depicted in Figure 9), and 392 control points with 160 elements are considered (Fig. 10). Given that $\mathrm{C}^{1}$ continuity is required in the S-FSDT, the bending strip method proposed by Kiendl et al. [46] is applied to maintain $\mathrm{C}^{1}$ continuity between patches. The stiffness of the bending strip stiffness is set as $E_{s}=10^{8} \mathrm{~Pa}$ in this paper. The normalized natural frequency $\omega^{*}=\left(\omega^{2} \rho h a^{4} / D\right)^{4}$ results obtained by the proposed method are listed in Table 14 for simply supported and clamped boundary condition, where $D=E h^{3} /(12(1-v))$ is the flexural rigidity of the plates. In addition to the FSDT-based IGA results, some available results obtained by Kirchhoff on the IGA [47], MKI method [48], EFG method [49], and node-based smoothing RPIM (NS-RPIM) method [50] are also presented for comparison purposes. The S-FSDT-based IGA results are in good agreement with the reference solutions for all considered boundary conditions.

\subsection{Buckling analysis}

The buckling load of a homogeneous rectangular plate with length $a$, width $b$, and thickness $h$ is computed. The pre-buckling forces can be obtained by using the equilibrium conditions expressed as follows:

$$
\sigma_{x}^{0}=\varsigma_{1} P_{1}, \quad \sigma_{y}^{0}=\varsigma_{2} P_{1}, \quad \tau_{x y}^{0}=0,
$$


where $P_{1}$ is the force per unit length, and $\varsigma_{1}$ and $\varsigma_{2}$ are the load parameters that indicate the

loading conditions. Negative values indicate that the plate is subjected to biaxial compressive loads, whereas positive values are used for tensile loads (Fig. 11).

The buckling load is normalized with $\bar{N}_{c r}=\frac{12 N_{c r} a^{2}\left(1-v^{2}\right)}{E h^{3}}$. Table 15 presents the normalized buckling loads for the homogeneous rectangular plate with different boundary conditions and aspect ratios. The results obtained by the S-FSDT-based IGA are in excellent agreement with the Levy solutions based on Kirchhoff theory [51, 52].

We also study the buckling response of an $\mathrm{Al} / \mathrm{Al}_{2} \mathrm{O}_{3}$ plate with different boundary conditions and aspect ratios. In this study, we set $b / h=0.01$. The buckling load $(M N / m)$ is presented in Table 16 for an $\mathrm{Al} / \mathrm{Al}_{2} \mathrm{O}_{3}$ plate with different boundary conditions and some gradient indexes. The results derived from the proposed method are in good agreement with the Levy solutions based on Kirchhoff theory [52]. Increasing the gradient index decreases the buckling load. The buckling load is increased from SFSF to SSSS and SCSC.

\section{Conclusions}

We presented a new locking-free plate formulation by using the characteristics of the NURBS-based IGA in combination with the S-FSDT theory for the study of homogeneous and nonhomogeneous functionally graded plates. Numerical examples for static bending, buckling and free vibration analysis were considered and their results were presented and discussed in detail. Aspects of the boundary conditions, gradient index, and geometric shape were also investigated. Our conclusions are as follows:

- the approach can be applied to thick and thin plates without special numerical techniques.

- one unknown is saved compared with the FSDT.

- the higher-order continuity of the IGA means that the potentially awkward $\mathrm{C}^{1}$-continuity required by the S-FSDT can be satisfied easily.

- the geometry can be described exactly, and arbitrary order continuity can be achieved, due to the inherent properties of the IGA framework.

- the new method can be implemented by researchers within existing open-source IGA codes with very little effort. 
We find that the S-FSDT-based IGA is well suited to the analysis of functionally graded plates.

The results show the robustness and accuracy of the S-FSDT-based IGA for both thin and thick plates. In this study we focused on the linear analysis of FGM plates. However, the method we propose is general; thus, it can be extended to other problems such as nonlinear analysis of plates. We also restricted our numerical study to the relatively simple geometries such as square, rectangular, circular, and multi-patch plates in order to compare with other authors, but in combination with techniques such as trimmed surfaces [53] or the finite cell method [54], the method proposed here could be extended to highly complex structures.

\section{Acknowledgements}

This work was supported by Jiangsu Province Graduate Students Research and Innovation Plan (Grant No. CXZZ13_0235) and the National Natural Science Foundation of China (Grant No. 51179063). Jack S. Hale was supported by the Fonds National de la Recherche, Luxembourg under the AFR Marie Curie COFUND scheme and partially supported by the University of Luxembourg. Tinh Quoc Bui (ID No. P14055) was supported by the Grant-in-Aid for Scientific Research (No. 26-04055) - Japan Society for Promotion of Science (JSPS). Stephane P. A. Bordas was partially supported by the European Research Council Starting Independent Research Grant (ERC Stg grant agreement No. 279578) entitled "Towards real time multiscale simulation of cutting in non-linear materials with applications to surgical simulation and computer guided surgery". 


\section{References}

[1] Jha DK, Kant T, Singh RK. A critical review of recent research on functionally graded plates. Compos Struct 2013;96:833-849.

[2] Liew KM, Zhao X, Ferreira AJM. A review of meshless methods for laminated and functionally graded plates and shells. Compos Struct 2011;93(8):2031-2041.

[3] Reissner E. The effect of transverse shear deformation on the bending of elastic plates. J. Appl. Mech 1945; 12, A69.

[4] Bathe KJ, Brezzi F, Cho SW. The MITC7 and MITC9 Plate bending elements. Comput Struct 1989;32(3-4):797-814.

[5] Hughes TJR, Cottrell JA, Bazilevs Y. Isogeometric analysis: CAD, finite elements, NURBS, exact geometry and mesh refinement. Comput Methods Appl Mech Eng 2005;194(39-41):4135-4195.

[6] Krysl P, Belytschko T. Analysis of thin plates by the element-free Galerkin method. Comput Mech 1995;17(1-2):26-35.

[7] Neves AMA, Ferreira AJM, Carrera E, Cinefra M, Roque CMC, Jorge RMN, Soares CMM. Static, free vibration and buckling analysis of isotropic and sandwich functionally graded plates using a quasi-3D higher-order shear deformation theory and a meshless technique. Compos Part B: Eng 2013;44(1):657-674.

[8] Thai HT, Choi DH. A simple first-order shear deformation theory for the bending and free vibration analysis of functionally graded plates. Compos Struct 2013;101:332-340.

[9] Thai HT, Choi DH. A simple first-order shear deformation theory for laminated composite plates. Compos Struct 2013;106:754-763.

[10] Brezzi F, Evans JA, Hughes TJR, Marini LD. New rectangular plate elements based on twist-Kirchhoff theory. Comput Methods Appl Mech Eng 2011;200(33-36):2547-2561.

[11] Cho JY, Atluri SN. Analysis of shear flexible beams, using the meshless local Petrov-Galerkin method, based on a locking-free formulation. Eng Computation. 2001;18(1/2):215-240.

[12] Tiago C, Leitao VMA. Eliminating shear-locking in meshless methods: a critical overview and a new framework for structural theories. In: Leitao VMA, Alves C, Armando Duarte C, editors. Advances in meshfree techniques. Computational methods in applied sciences, vol. 5. Berlin: 
Springer; 2007. p. 123-45.

[13] Cottrell JA, Reali A, Bazilevs Y, Hughes TJR. Isogeometric analysis of structural vibrations. Comput Methods Appl Mech Eng 2006;195(41-43):5257-5296.

[14] Valizadeh N, Natarajan S, Gonzalez-Estrada OA, Rabczuk T, Bui TQ, Bordas SPA. NURBS-based finite element analysis of functionally graded plates: Static bending, vibration, buckling and flutter. Compos Struct 2013;99:309-326.

[15] Tran LV, Ferreira AJM, Nguyen-Xuan H. Isogeometric analysis of functionally graded plates using higher-order shear deformation theory. Compos Part B: Eng 2013;51:368-383.

[16] Valizadeh N, Bui TQ, Vu VT, Thai HT, Nguyen MN. isogeometric simulation for buckling, free and forced vibration of orthotropic plates. Int. J. Appl. Mechanics 2013;05(02):1350017.

[17] Kiendl J, Bletzinger KU, Linhard J, Wüchner R. Isogeometric shell analysis with Kirchhoff-Love elements. Comput Methods Appl Mech Eng 2009;198(49-52):3902-3914.

[18] Benson DJ, Bazilevs Y, Hsu MC, Hughes TJR. Isogeometric shell analysis: The Reissner-Mindlin shell. Comput Methods Appl Mech Eng 2010;199(5-8):276-289.

[19] Shojaee S, Valizadeh N, Izadpanah E, Bui TQ, Vu TV. Free vibration and buckling analysis of laminated composite plates using the NURBS-based isogeometric finite element method. Compos Struct 2012;94(5):1677-1693.

[20] Bazilevs Y, Calo VM, Cottrell JA, Hughes TJR, Reali A, Scovazzi G. Variational multiscale residual-based turbulence modeling for large eddy simulation of incompressible flows. Comput Methods Appl Mech Eng 2007;197(1-4):173-201.

[21] Bazilevs Y, Calo VM, Hughes TJR, Zhang Y. Isogeometric fluid-structure interaction: theory, algorithms, and computations. Comput Mech 2008;43(1):3-37.

[22] Verhoosel CV, Scott MA, Hughes TJR, de Borst R. An isogeometric analysis approach to gradient damage models. Int J Numer Meth Eng 2011;86(1):115-134.

[23] Wall WA, Frenzel MA, Cyron C. Isogeometric structural shape optimization. Comput Methods Appl Mech Eng 2008;197(33-40):2976-2988.

[24] Echter R, Bischoff M. Numerical efficiency, locking and unlocking of NURBS finite elements. Comput Methods Appl Mech Eng 2010;199(5-8):374-382.

[25] da Veiga LB, Buffa A, Lovadina C, Martinelli M, Sangalli G. An isogeometric method for the Reissner-Mindlin plate bending problem. Comput Methods Appl Mech Eng 2012;209-212:45-53. 
[26] Nguyen VP, Bordas SPA, Rabczuk T. Isogeometric analysis: an overview and computer implementation aspects. arXiv:1205.2129 [cs, math] (2012). at $<$ http://arxiv.org/abs/1205.2129>.

[27]de Falco C, Reali A, Vázquez R. GeoPDEs: A research tool for Isogeometric Analysis of PDEs. Adv Eng Softw 2011;42(12):1020-1034.

[28] Arnold D, Falk R. The Boundary Layer for the Reissner-Mindlin Plate Model. Siam J Math Anal 1990;21(2):281-312.

[29] Piegl L, Tiller W. The NURBS Book. Springer Berlin Heidelberg, 1995.

[30] Zienkiewicz OC, Xu Z, Zeng LF, Samuelsson A, Wiberg N. Linked interpolation for Reissner-Mindlin plate elements: Part I-A simple quadrilateral. Int J Numer Meth Eng $1993 ; 36(18): 3043-3056$.

[31] Neves AMA, Ferreira AJM, Carrera E, Cinefra M, Roque CMC, Jorge RMN, Soares CMM. A quasi-3D hyperbolic shear deformation theory for the static and free vibration analysis of functionally graded plates. Compos Struct 2012;94(5):1814-1825.

[32] Carrera E, Brischetto S, Cinefra M, Soave M. Effects of thickness stretching in functionally graded plates and shells. Compos Part B: Eng 2011;42(2):123-133.

[33] Lee YY, Zhao X, Liew KM. Thermoelastic analysis of functionally graded plates using the element-free kp-Ritz method. Smart Mater. Struct 2009;18(3):35007.

[34] Nguyen-Xuan H, Tran LV, Nguyen-Thoi T, Vu-Do HC. Analysis of functionally graded plates using an edge-based smoothed finite element method. Compos Struct 2011;93(11):3019-3039.

[35] Liu GR. Mesh free methods: moving beyond the finite element method. CRC Press, 2003.

[36] Li XY, Ding HJ, Chen WQ. Elasticity solutions for a transversely isotropic functionally graded circular plate subject to an axisymmetric transverse load qrk. Int J Solids Struct 2008;45(1):191-210.

[37] Reddy JN, Wang CM, Kitipornchai S. Axisymmetric bending of functionally graded circular and annular plates. Euro J Mechan A/Solids 1999;18(2):185-199.

[38] Matsunaga $\mathrm{H}$. Free vibration and stability of functionally graded plates according to a 2-D higher-order deformation theory. Compos Struct 2008;82(4):499-512.

[39] Thai HT, Kim SE. A simple higher-order shear deformation theory for bending and free vibration analysis of functionally graded plates. Compos Struct 2013;96:165-173.

[40] Thai HT, Choi DH. Finite element formulation of various four unknown shear deformation 
theories for functionally graded plates. Finite Elem Anal Des 2013;75:50-61.

[41] Zhao X, Lee YY, Liew KM. Free vibration analysis of functionally graded plates using the element-free kp-Ritz method. J Sound Vib 2009;319(3-5):918-939.

[42] Yin SH, Yu TT, Liu P. Free Vibration Analyses of FGM Thin Plates by Isogeometric Analysis Based on Classical Plate Theory and Physical Neutral Surface. Advances in Mechanical Engineering 2013; Volume 2013, Article ID 634584, 10 pages.

[43] Baferani AH, Saidi AR, Jomehzadeh E. An exact solution for free vibration of thin functionally graded rectangular plates. Proc Inst Mech Eng Part C J Mech Eng Sci 2011;225:(C3):526-536.

[44] Hosseini-Hashemi S, Fadaee M, Es'Haghi M. A novel approach for in-plane/out-of-plane frequency analysis of functionally graded circular/annular plates. Int $\mathrm{J}$ Mech Sci 2010;52(8):1025-1035.

[45] Ebrahimi F, Rastgoo A, Atai AA. A theoretical analysis of smart moderately thick shear deformable annular functionally graded plate. Euro J Mechan A/Solids 2009;28(5):962-973.

[46] Kiendl J, Bazilevs Y, Hsu MC, Wüchner R, Bletzinger KU. The bending strip method for isogeometric analysis of Kirchhoff-Love shell structures comprised of multiple patches. Comput Methods Appl Mech Eng 2010;199(37-40):2403-2416.

[47] Shojaee S, Izadpanah E, Valizadeh N, Kiendl J. Free vibration analysis of thin plates by using a NURBS-based isogeometric approach. Finite Elem Anal Des 2012;61:23-34.

[48] Bui TQ, Nguyen MN. A moving Kriging interpolation-based meshfree method for free vibration analysis of Kirchhoff plates. Comput Struct 2011;89(3-4):380-394.

[49] Liu GR, Chen XL. A mesh-free method for static and free vibration analyses of thin plates of complicated shape. J Sound Vib 2001;241(5):839-855.

[50] Cui XY, Liu GR, Li GY, Zhang GY. A thin plate formulation without rotation DOFs based on the radial point interpolation method and triangular cells. Int $\mathrm{J}$ Numer Meth Eng 2011;85(8):958-986.

[51] Yu LH, Wang CY. Buckling of rectangular plates on an elastic foundation using the Levy method. AIAA J 2008;46(12):3163-6.

[52] Mohammadi M, Saidi A, Jomehzadeh E. Levy Solution for Buckling Analysis of Functionally Graded Rectangular Plates. Appl Compos Mater 2010;17(2):81-93.

[53] Kim HJ, Seo YD, Youn SK. Isogeometric analysis for trimmed CAD surfaces. Comput Methods Appl Mech Eng 2009;198(37-40):2982-2995. 
[54] Schillinger D, Ruess M, Zander N, Bazilevs Y, Düster A, Rank E. Small and large deformation analysis with the p- and B-spline versions of the Finite Cell Method. Comput Mech 2012;50(4):445-478. 


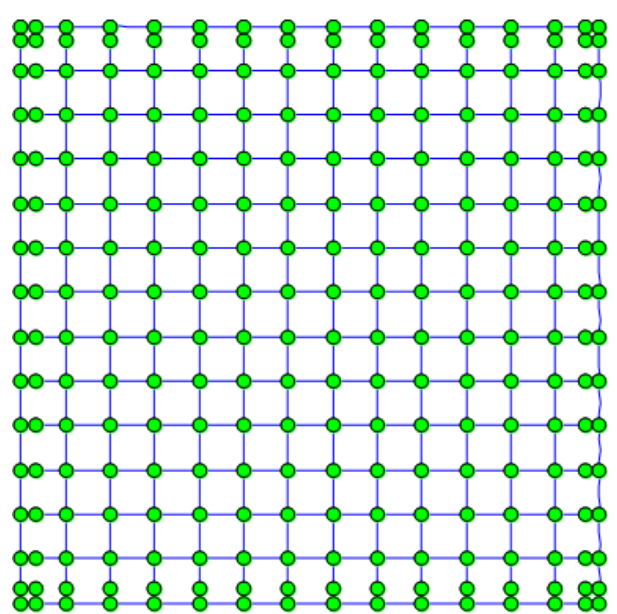

(a)

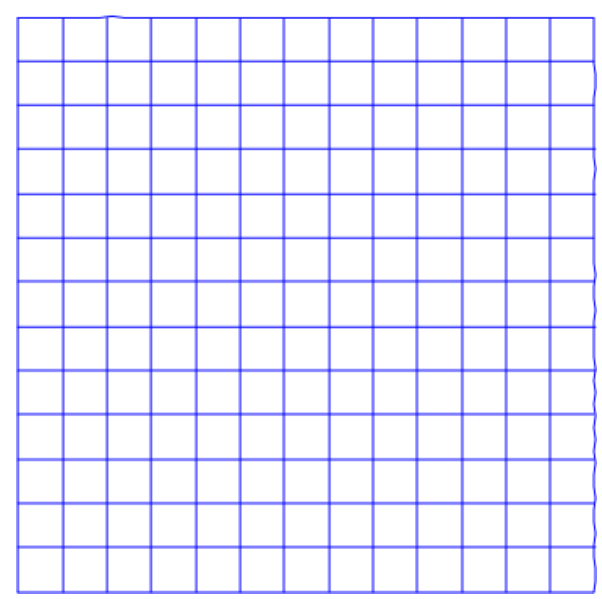

(b)

Fig. 1. A square plate with $16 \times 16$ control points and $13 \times 13$ elements by using cubic NURBS basis function: (a) control mesh and (b) physical mesh. 
Table 1. Comparisons of the normalized central deflection obtained by the IGA for the S-FSDT, FSDT, and analytical method [30].

(a) Fully simply supported

\begin{tabular}{|c|c|c|c|c|c|c|c|}
\hline$a / h$ & Method & $\bar{w}$ & Error $(\%)$ & $a / h$ & Method & $\bar{w}$ & $\begin{array}{l}\text { Error } \\
(\%)\end{array}$ \\
\hline \multirow{5}{*}{20} & FSDT-Quadratic & 0.4114 & 0.1461 & \multirow{5}{*}{100} & FSDT -Quadratic & 0.4048 & 0.3937 \\
\hline & S-FSDT-Quadratic & 0.4109 & 0.0243 & & S-FSDT-Quadratic & 0.4058 & 0.1476 \\
\hline & FSDT-Cubic & 0.4115 & 0.1704 & & FSDT-Cubic & 0.4064 & 0 \\
\hline & S-FSDT-Cubic & 0.4115 & 0.1704 & & S-FSDT-Cubic & 0.4064 & 0 \\
\hline & Exact [30] & 0.4108 & & & Exact [30] & 0.4064 & \\
\hline \multirow{5}{*}{$10^{3}$} & FSDT-Quadratic & 0.3654 & 10.0443 & \multirow{5}{*}{$10^{4}$} & FSDT-Quadratic & 0.3552 & 12.5554 \\
\hline & S-FSDT-Quadratic & 0.4056 & 0.1477 & & S-FSDT-Quadratic & 0.4056 & 0.1477 \\
\hline & FSDT-Cubic & 0.4069 & 0.1723 & & FSDT-Cubic & 0.3825 & 5.8346 \\
\hline & S-FSDT-Cubic & 0.4062 & 0 & & S-FSDT-Cubic & 0.4062 & 0 \\
\hline & Exact [30] & 0.4062 & & & Exact [30] & 0.4062 & \\
\hline \multirow{5}{*}{$10^{5}$} & FSDT-Quadratic & 0.3551 & 12.58 & \multirow{5}{*}{$10^{6}$} & FSDT-Quadratic & 0.3551 & 12.58 \\
\hline & S-FSDT-Quadratic & 0.4056 & 0.1477 & & S-FSDT-Quadratic & 0.4056 & 0.1477 \\
\hline & FSDT-Cubic & 0.3556 & 12.4569 & & FSDT-Cubic & 0.3551 & 12.58 \\
\hline & S-FSDT-Cubic & 0.4062 & 0 & & S-FSDT-Cubic & 0.4062 & 0 \\
\hline & Exact [30] & 0.4062 & & & Exact [30] & 0.4062 & \\
\hline
\end{tabular}

(b) Fully clamped

\begin{tabular}{|c|c|c|c|c|c|c|c|}
\hline$a / h$ & Method & $\bar{w}$ & Error $(\%)$ & $a / h$ & Method & $\bar{w}$ & Error $(\%)$ \\
\hline \multirow{4}{*}{100} & FSDT-Quadratic & 0.1213 & 4.1107 & \multirow{4}{*}{$10^{3}$} & FSDT-Quadratic & 0.0252 & 80.0791 \\
\hline & S-FSDT-Quadratic & 0.1244 & 1.6601 & & S-FSDT-Quadratic & 0.1242 & 1.8182 \\
\hline & FSDT-Cubic & 0.1268 & 0.2372 & & FSDT-Cubic & 0.1268 & 0.2372 \\
\hline & S-FSDT-Cubic & 0.1267 & 0.1581 & & S-FSDT-Cubic & 0.1265 & 0 \\
\hline
\end{tabular}




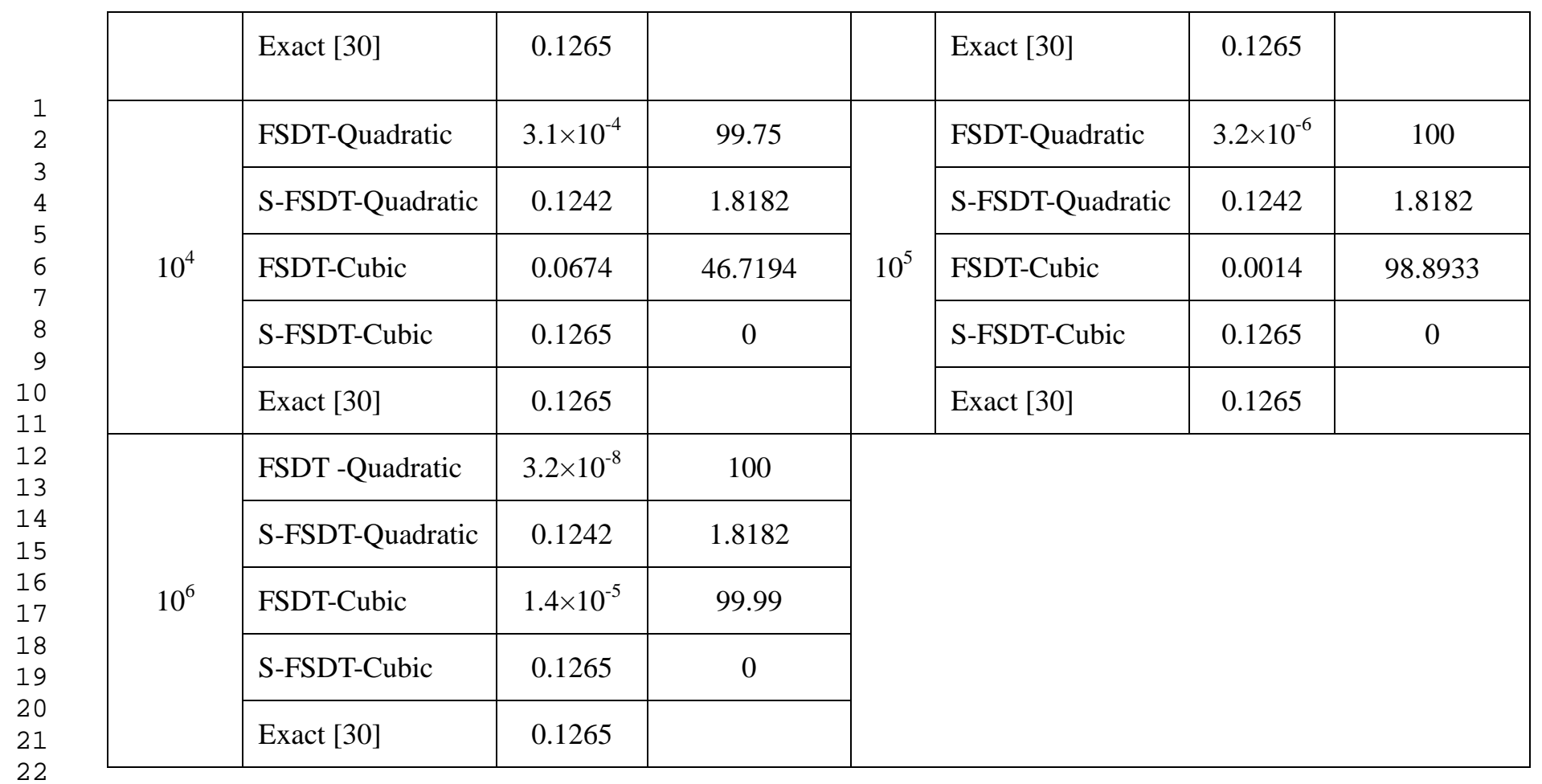


Table 2. Comparisons of the normalized central deflection, bending deflections, and shear deflections obtained by the IGA based on the S-FSDT for a fully simply supported square plate.

\begin{tabular}{|c|c|c|c|}
\hline$a / h$ & $\bar{w}$ & $\bar{w}_{b}$ & $\bar{w}_{s}$ \\
\hline 5 & 0.490431 & 0.406235 & 0.084196 \\
\hline 10 & 0.427284 & 0.406235 & 0.021049 \\
\hline 20 & 0.411497 & 0.406235 & 0.005262 \\
\hline 50 & 0.407077 & 0.406235 & $8.42 \times 10^{-4}$ \\
\hline 100 & 0.406446 & 0.406235 & $2.10 \times 10^{-4}$ \\
\hline $10^{3}$ & 0.406237 & 0.406235 & $2.10 \times 10^{-6}$ \\
\hline $10^{4}$ & 0.406235 & 0.406235 & $2.10 \times 10^{-8}$ \\
\hline $10^{5}$ & 0.406235 & 0.406235 & $2.10 \times 10^{-10}$ \\
\hline
\end{tabular}




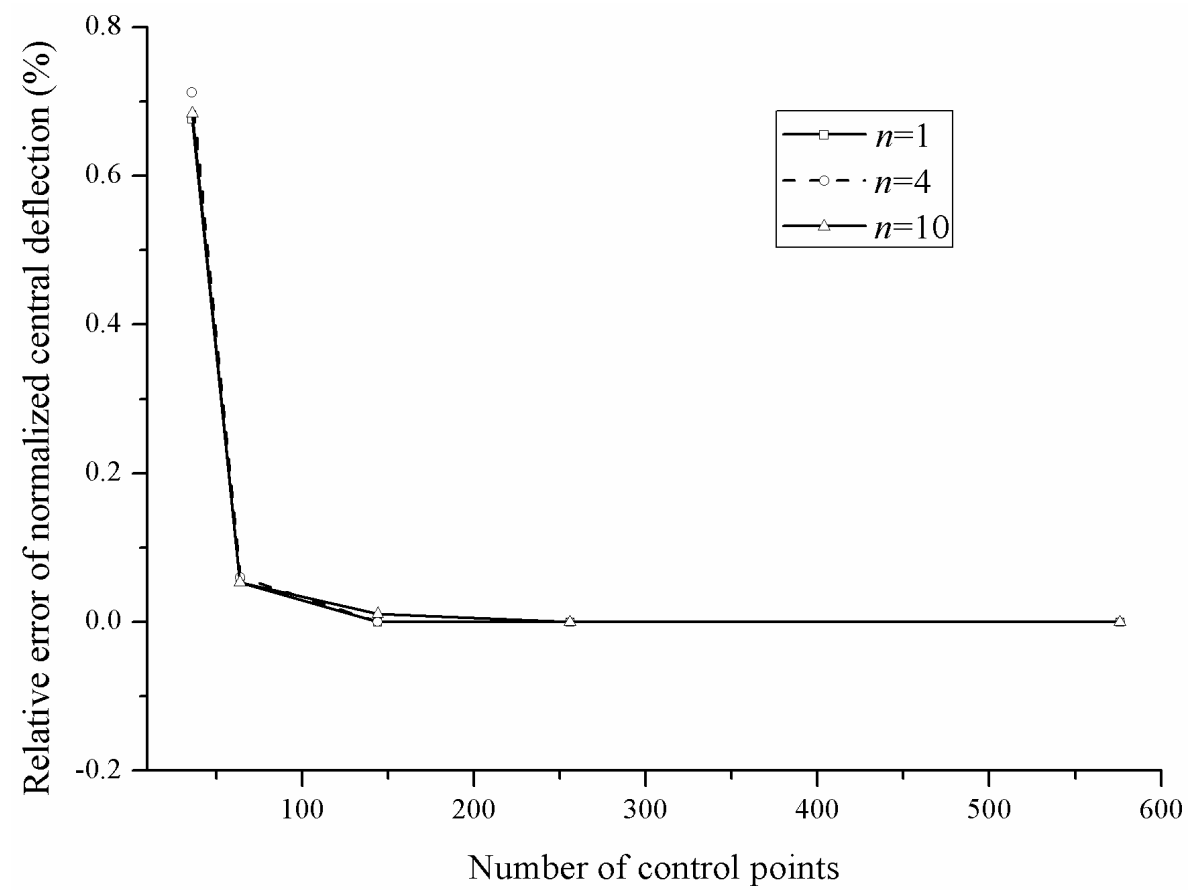

Fig. 2. Convergence results in the relative error of normalized central deflection. 
Table 3. Normalized central deflections of $\mathrm{Al} / \mathrm{Al}_{2} \mathrm{O}_{3}$ square plate under sinusoidal loads.

\begin{tabular}{|c|c|c|c|c|c|}
\hline & Method & Number of control points & $n=1$ & $n=4$ & $n=10$ \\
\hline \multirow{5}{*}{\multicolumn{2}{|c|}{ S-FSDT based IGA }} & $6 \times 6$ & 0.5587 & 0.8227 & 0.9297 \\
\hline & & $8 \times 8$ & 0.5622 & 0.8281 & 0.9356 \\
\hline & & $12 \times 12$ & 0.5625 & 0.8286 & 0.9360 \\
\hline & & $16 \times 16$ & 0.5625 & 0.8286 & 0.9361 \\
\hline & & $24 \times 24$ & 0.5625 & 0.8286 & 0.9361 \\
\hline \multicolumn{2}{|r|}{ FSDT based IGA } & $16 \times 16$ & 0.5625 & 0.8286 & 0.9361 \\
\hline \multirow{3}{*}{ Exact } & quasi-3D hyperbolic [31] & - & 0.5648 & 0.8241 & 0.9228 \\
\hline & Higher-order theory [32] & - & 0.5625 & 0.8286 & 0.9361 \\
\hline & S-FSDT [8] & - & 0.5625 & 0.8286 & 0.9361 \\
\hline
\end{tabular}

Table 4. Normalized central deflections of $\mathrm{Al} / \mathrm{Al}_{2} \mathrm{O}_{3}$ square plates under sinusoidal loads.

\begin{tabular}{|c|c|c|c|c|c|c|c|c|c|c|}
\hline & \multirow{2}{*}{ Method } & \multicolumn{3}{|c|}{$a / h=100$} & \multicolumn{3}{|c|}{$a / h=10$} & \multicolumn{3}{|c|}{$a / h=4$} \\
\hline & & $n=1$ & $n=4$ & $n=10$ & $n=1$ & $n=4$ & $n=10$ & $n=1$ & $n=4$ & $n=10$ \\
\hline \multicolumn{2}{|c|}{ S-FSDT based IGA } & 0.5625 & 0.8286 & 0.9361 & 0.5890 & 0.8736 & 0.9966 & 0.7291 & 1.1125 & 1.3178 \\
\hline \multicolumn{2}{|c|}{ FSDT based IGA } & 0.5625 & 0.8286 & 0.9361 & 0.5890 & 0.8736 & 0.9966 & 0.7291 & 1.1125 & 1.3178 \\
\hline \multirow{3}{*}{ Exact } & $\begin{array}{c}\text { quasi-3D } \\
\text { hyperbolic [31] }\end{array}$ & 0.5648 & 0.8241 & 0.9228 & 0.5868 & 0.8698 & 0.9886 & 0.702 & 1.1095 & 1.3327 \\
\hline & $\begin{array}{l}\text { Higher-order } \\
\text { theory [32] }\end{array}$ & 0.5625 & 0.8286 & 0.9361 & 0.5875 & 0.8821 & 1.0072 & 0.7171 & 1.1585 & 1.3745 \\
\hline & S-FSDT [8] & 0.5625 & 0.8286 & 0.9361 & 0.5890 & 0.8736 & 0.9966 & 0.7291 & 1.1125 & 1.3178 \\
\hline
\end{tabular}


Table 5. Normalized deflection of $\mathrm{Al} / \mathrm{ZrO}_{2}-1$ plate with different length-thickness ratios $a / h$ and gradient indexes $n$.

\begin{tabular}{|c|c|c|c|c|c|c|}
\hline Type & $a / h$ & Method & $n=0$ & $n=0.5$ & $n=1$ & $n=2$ \\
\hline \multirow{8}{*}{ SSSS } & \multirow{4}{*}{5} & S-FSDT based IGA & 0.1717 & 0.2324 & 0.2719 & 0.3115 \\
\hline & & FSDT based IGA & 0.1717 & 0.2324 & 0.2719 & 0.3115 \\
\hline & & FSDT based kp-Ritz [33] & 0.1722 & 0.2403 & 0.2811 & 0.3221 \\
\hline & & FSDT based ES-DSG3 [34] & 0.1703 & 0.2232 & 0.2522 & 0.2827 \\
\hline & \multirow{2}{*}{20} & S-FSDT based IGA & 0.1440 & 0.1972 & 0.2310 & 0.2628 \\
\hline & & FSDT based IGA & 0.1440 & 0.1972 & 0.2310 & 0.2628 \\
\hline & \multirow{2}{*}{100} & S-FSDT based IGA & 0.1423 & 0.1949 & 0.2284 & 0.2597 \\
\hline & & FSDT based IGA & 0.1423 & 0.1949 & 0.2284 & 0.2597 \\
\hline \multirow{6}{*}{ SFSS } & \multirow{2}{*}{5} & S-FSDT based IGA & 0.3164 & 0.4299 & 0.5032 & 0.5752 \\
\hline & & FSDT based IGA & 0.3175 & 0.4314 & 0.5049 & 0.5772 \\
\hline & \multirow{2}{*}{20} & S-FSDT based IGA & 0.2800 & 0.3835 & 0.4493 & 0.5109 \\
\hline & & FSDT based IGA & 0.2803 & 0.3838 & 0.4497 & 0.5114 \\
\hline & \multirow{2}{*}{100} & S-FSDT based IGA & 0.2777 & 0.3805 & 0.4458 & 0.5068 \\
\hline & & FSDT based IGA & 0.2777 & 0.3805 & 0.4458 & 0.5069 \\
\hline \multirow{6}{*}{ SFSF } & \multirow{2}{*}{5} & S-FSDT based IGA & 0.5083 & 0.6918 & 0.8099 & 0.9247 \\
\hline & & FSDT based IGA & 0.5089 & 0.6926 & 0.8108 & 0.9258 \\
\hline & \multirow{2}{*}{20} & S-FSDT based IGA & 0.4614 & 0.6319 & 0.7404 & 0.8420 \\
\hline & & FSDT based IGA & 0.4615 & 0.6321 & 0.7406 & 0.8422 \\
\hline & \multirow{2}{*}{100} & S-FSDT based IGA & 0.4584 & 0.6281 & 0.7360 & 0.8367 \\
\hline & & FSDT based IGA & 0.4584 & 0.6281 & 0.7360 & 0.8367 \\
\hline
\end{tabular}


Table 6. Normalized deflection of $\mathrm{Al} / \mathrm{Al}_{2} \mathrm{O}_{3}$ thin plate under different gradient indexes and boundary conditions

\begin{tabular}{|c|c|c|c|c|c|c|c|}
\hline \multirow{2}{*}{ Type } & Method & $n=0$ & $n=0.5$ & $n=1$ & $n=2$ & $n=5$ & $n=10$ \\
\hline \multirow{2}{*}{ SSSS } & S-FSDT & 0.4438 & 0.6846 & 0.8904 & 1.1411 & 1.3494 & 1.4816 \\
\cline { 2 - 8 } & FSDT & 0.4438 & 0.6847 & 0.8904 & 1.1411 & 1.3494 & 1.4816 \\
\hline \multirow{2}{*}{ SFSF } & S-FSDT & 1.4302 & 2.2062 & 2.8692 & 3.6770 & 4.3483 & 4.7740 \\
\cline { 2 - 8 } & FSDT & 1.4302 & 2.2062 & 2.8693 & 3.6770 & 4.3483 & 4.7740 \\
\hline \multirow{2}{*}{ SCSC } & S-FSDT & 0.2096 & 0.3232 & 0.4204 & 0.5387 & 0.6372 & 0.6996 \\
\cline { 2 - 8 } & FSDT & 0.2097 & 0.3234 & 0.4205 & 0.5389 & 0.6375 & 0.7000 \\
\hline \multirow{2}{*}{ CCCC } & S-FSDT & 0.1384 & 0.2135 & 0.2776 & 0.3557 & 0.4208 & 0.4621 \\
\cline { 2 - 8 } & FSDT & 0.1384 & 0.2135 & 0.2776 & 0.3558 & 0.4209 & 0.4622 \\
\hline
\end{tabular}




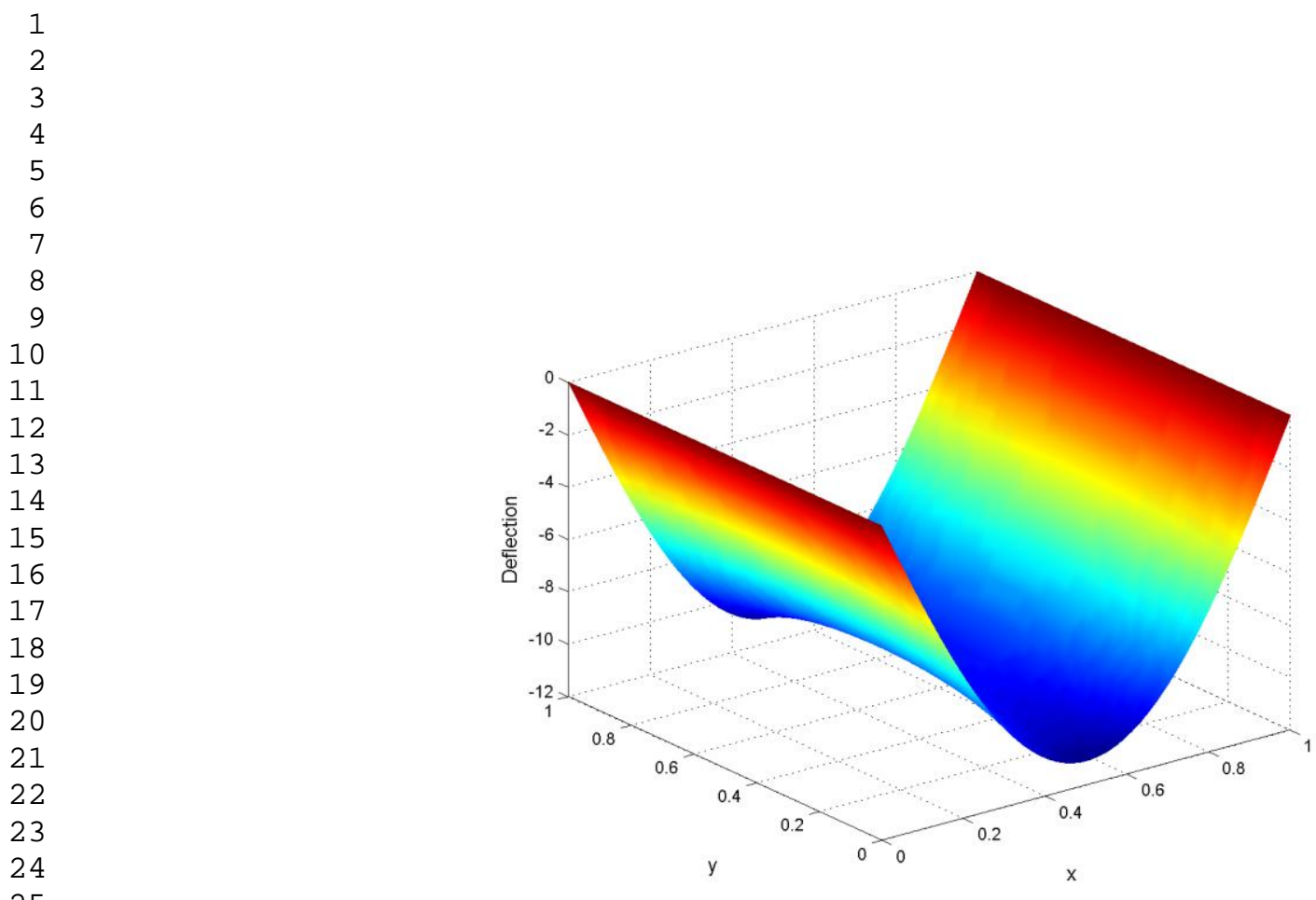

(a)

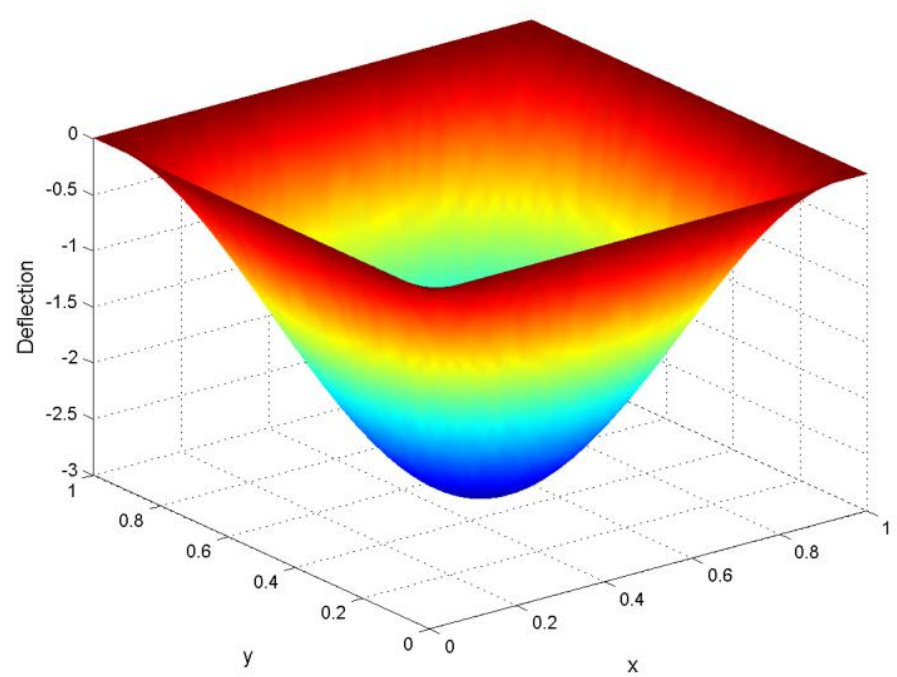

(b) 


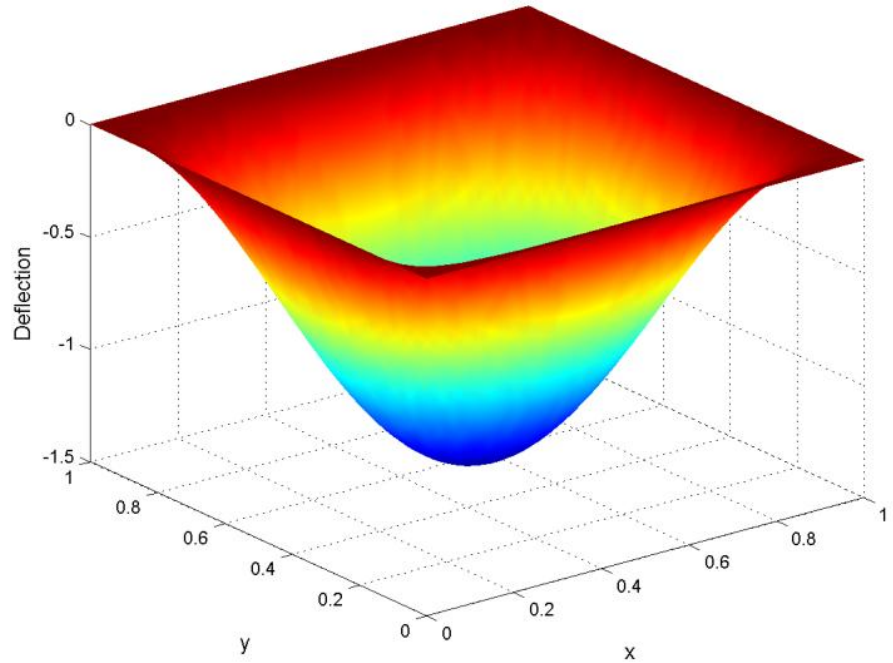

(c)

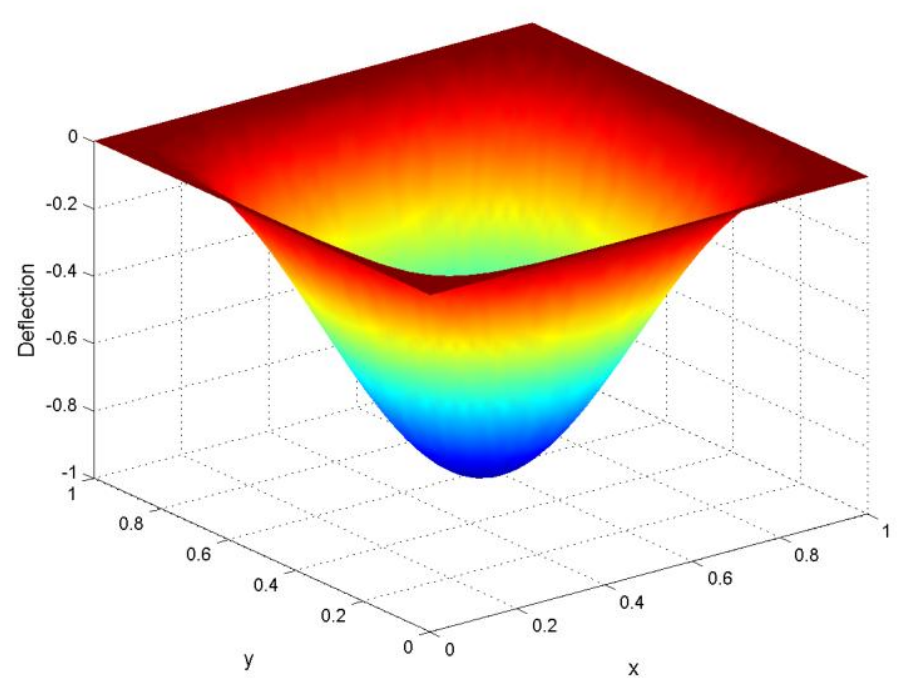

(d)

Fig. 3. Deflection of $\mathrm{Al} / \mathrm{Al}_{2} \mathrm{O}_{3}$ thin plate with different boundary conditions $(n=2)$ : (a) SFSF, (b) SSSS, (c) SCSC, and (d) CCCC. 
Table 7. Normalized deflection for a circular plate subjected to a uniformly distributed pressure.

\begin{tabular}{|c|c|c|c|c|c|c|c|}
\hline \multirow[b]{2}{*}{$\begin{array}{l}\text { Boundary } \\
\text { condition }\end{array}$} & \multirow[b]{2}{*}{$\begin{array}{l}\text { Exact } \\
\text { [35] }\end{array}$} & \multirow[b]{2}{*}{$\begin{array}{c}\text { FSDT based } \\
\text { IGA }\end{array}$} & \multirow[b]{2}{*}{$\begin{array}{c}\text { S-FSDT } \\
\text { based IGA }\end{array}$} & \multirow{2}{*}{$\begin{array}{l}\text { Kirchhoff } \\
\text { theory based } \\
\text { MLPG [35] }\end{array}$} & \multicolumn{3}{|c|}{ Error $(\%)$} \\
\hline & & & & & $\begin{array}{l}\text { FSDT } \\
\text { based } \\
\text { IGA }\end{array}$ & $\begin{array}{c}\text { S-FSDT } \\
\text { based } \\
\text { IGA }\end{array}$ & $\begin{array}{l}\text { Kirchhoff } \\
\text { theory based } \\
\text { MLPG [35] }\end{array}$ \\
\hline $\begin{array}{c}\text { Simply } \\
\text { supported }\end{array}$ & 0.0637 & 0.0637 & 0.0634 & 0.0639 & 0.0738 & 0.5196 & 0.3265 \\
\hline Clamped & 0.0156 & 0.0156 & 0.0156 & 0.0156 & 0.2430 & 0.0832 & 0.4160 \\
\hline
\end{tabular}

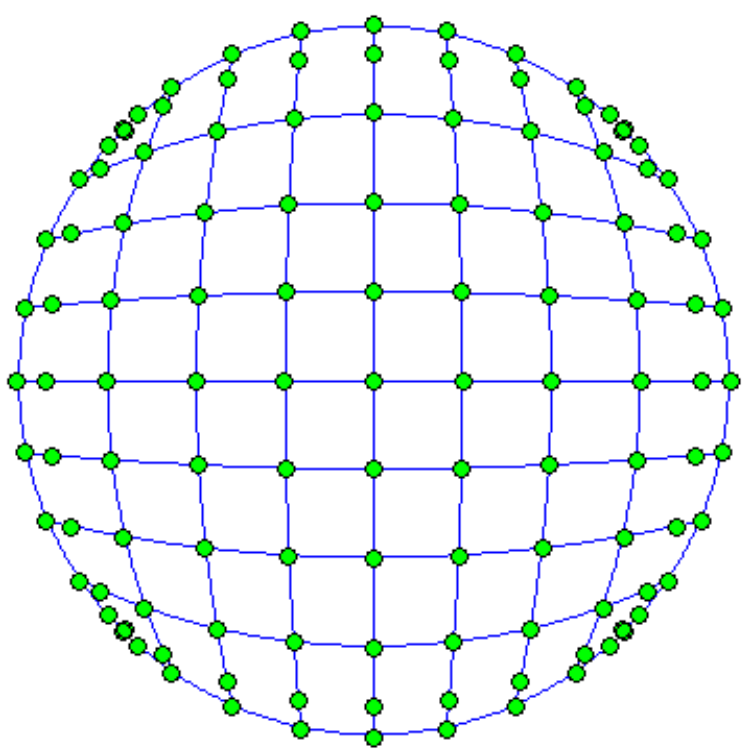

Fig. 4. Control points and physical mesh of a circular plate with cubic NURBS basis function. 
1

2

3

4

5

6

7

9

10

11

12

13

14

15

16

17

18

19

20

21

22

23

24

25

26

27

28

29

30

31

32

33

34

35

36

37

38

39

40

41

42

43

44

45

46

47

48

49

50

51

52

53

54

55

56

57

58

59

60

61

62

63

64

65

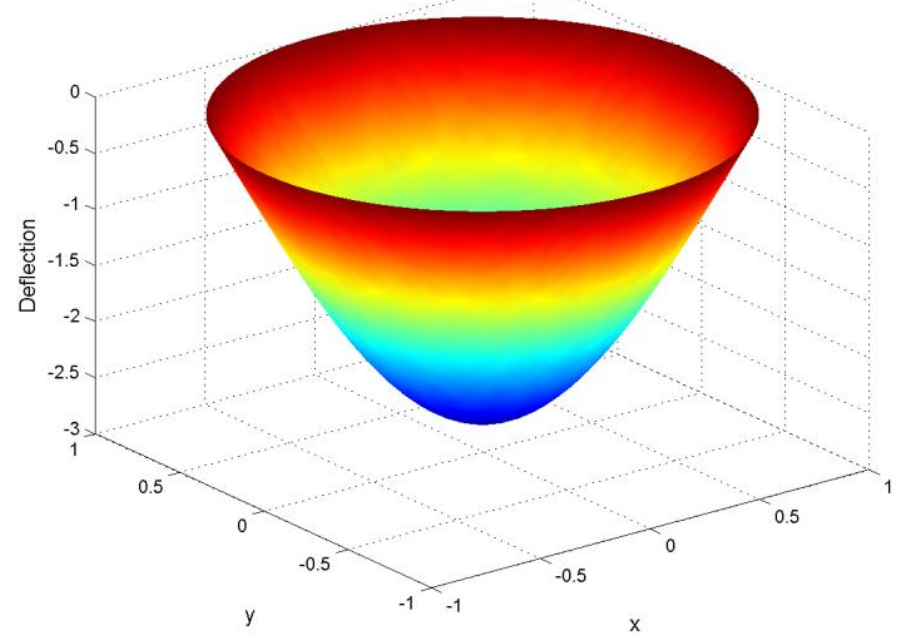

(a)

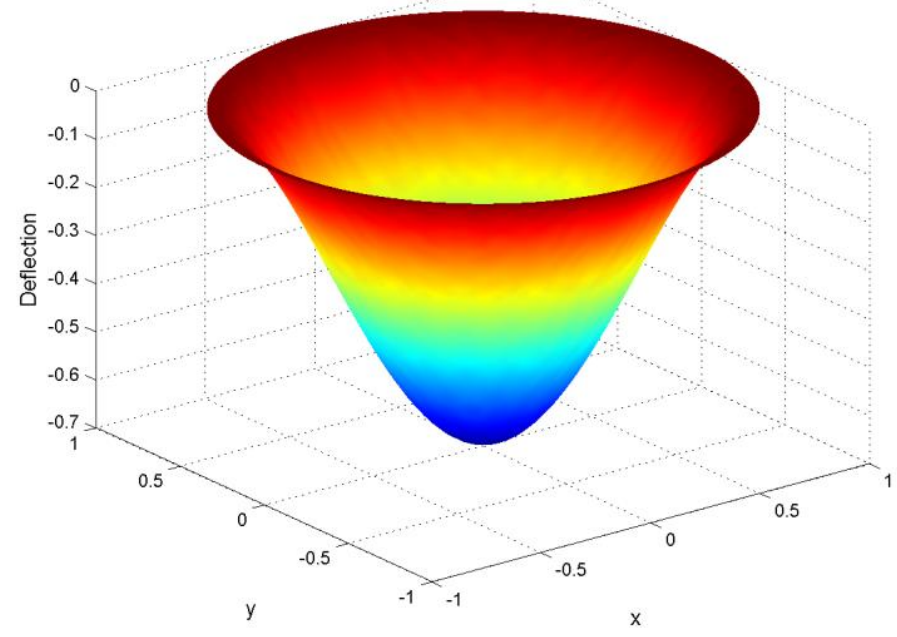

(b) clamped. 
Table 8. Normalized center deflection of circular titanium/zirconium plate subjected to a uniform load.

(a) Roller supported boundary condition

\begin{tabular}{|c|c|c|c|c|c|c|c|c|c|}
\hline$h / R$ & Method & $n=0$ & $n=2$ & $n=4$ & $n=8$ & $n=10$ & $n=50$ & $n=100$ & $n=10^{5}$ \\
\hline \multirow{5}{*}{0.05} & $\begin{array}{l}\text { Elasticity } \\
\text { solutions [36] }\end{array}$ & 10.3910 & 5.7090 & 5.2190 & 4.8090 & 4.7000 & 4.2560 & 4.1870 & 4.1180 \\
\hline & Reddy [37] & 10.3960 & 5.7140 & 5.2230 & 4.8120 & 4.7040 & 4.2580 & 4.1890 & 4.1180 \\
\hline & $\begin{array}{l}\text { HSDT based IGA } \\
{[15]}\end{array}$ & 10.2203 & 5.6100 & 5.1299 & 4.7299 & 4.6239 & 4.1881 & 4.1198 & 4.0495 \\
\hline & FSDT based IGA & 10.3963 & 5.7137 & 5.2227 & 4.8124 & 4.7036 & 4.2584 & 4.1895 & 4.1170 \\
\hline & $\begin{array}{l}\text { S-FSDT based } \\
\text { IGA }\end{array}$ & 10.3411 & 5.4851 & 5.0966 & 4.7513 & 4.6542 & 4.2347 & 4.1670 & 4.0951 \\
\hline \multirow{5}{*}{0.1} & $\begin{array}{l}\text { Elasticity } \\
\text { solutions [36] }\end{array}$ & 10.4600 & 5.7380 & 5.2450 & 4.8350 & 4.7270 & 4.2830 & 4.2150 & 4.1460 \\
\hline & Reddy [37] & 10.4810 & 5.7560 & 5.2610 & 4.8480 & 4.7390 & 4.2930 & 4.2230 & 4.1510 \\
\hline & $\begin{array}{l}\text { HSDT based IGA } \\
{[15]}\end{array}$ & 10.3440 & 5.6742 & 5.1879 & 4.7838 & 4.6769 & 4.2377 & 4.1690 & 4.0970 \\
\hline & FSDT based IGA & 10.4817 & 5.7561 & 5.2612 & 4.8487 & 4.7394 & 4.2927 & 4.2235 & 4.1508 \\
\hline & $\begin{array}{l}\text { S-FSDT based } \\
\text { IGA }\end{array}$ & 10.4262 & 5.5273 & 5.1349 & 4.7874 & 4.6899 & 4.2688 & 4.2009 & 4.1288 \\
\hline \multirow{5}{*}{0.2} & $\begin{array}{l}\text { Elasticity } \\
\text { solutions [36] }\end{array}$ & 10.7360 & 5.8530 & 5.3510 & 4.9410 & 4.8330 & 4.3920 & 4.3240 & 4.2550 \\
\hline & Reddy [37] & 10.8220 & 5.9250 & 5.4140 & 4.9930 & 4.8820 & 4.4290 & 4.3590 & 4.2860 \\
\hline & $\begin{array}{l}\text { HSDT based IGA } \\
{[15]}\end{array}$ & 10.6973 & 5.8475 & 5.3439 & 4.9315 & 4.8230 & 4.3793 & 4.3098 & 4.2369 \\
\hline & FSDT based IGA & 10.8223 & 5.9250 & 5.4147 & 4.9932 & 4.8821 & 4.4291 & 4.3592 & 4.2857 \\
\hline & $\begin{array}{l}\text { S-FSDT based } \\
\text { IGA }\end{array}$ & 10.7667 & 5.6961 & 5.2883 & 4.9319 & 4.8326 & 4.4052 & 4.3365 & 4.2637 \\
\hline
\end{tabular}

(b) Clamped boundary condition

\begin{tabular}{|c|l|c|c|c|c|c|c|c|c|}
\hline$h / R$ & \multicolumn{1}{|c|}{ Method } & $n=0$ & $n=2$ & $n=4$ & $n=8$ & $n=10$ & $n=50$ & $n=100$ & $n=10^{5}$ \\
\hline \multirow{5}{*}{0.05} & $\begin{array}{l}\text { Elasticity } \\
\text { solutions [36] }\end{array}$ & 2.561 & 1.4050 & 1.2840 & 1.1840 & 1.1570 & 1.0490 & 1.0320 & 1.0150 \\
\cline { 2 - 9 } & \begin{tabular}{l} 
Reddy [37] \\
\cline { 2 - 9 } \\
\cline { 2 - 9 }
\end{tabular} & 2.554 & 1.4020 & 1.2820 & 1.1810 & 1.1550 & 1.0460 & 1.0290 & 1.0110 \\
\cline { 2 - 9 } & FSDT based IGA & 2.548 & 1.3990 & 1.2786 & 1.1785 & 1.1520 & 1.0435 & 1.0267 & 1.0092 \\
\cline { 2 - 9 } & $\begin{array}{l}\text { S-FSDT based IGA } \\
\text { IGA }\end{array}$ & 2.5539 & 1.4024 & 1.2819 & 1.1814 & 1.1547 & 1.0459 & 1.0291 & 1.0114 \\
\hline
\end{tabular}




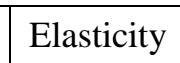

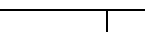

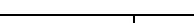

\begin{tabular}{|c|c|c|c|c|c|c|c|c|c|}
\hline \multirow{5}{*}{0.1} & $\begin{array}{l}\text { Elasticity } \\
\text { solutions [36] }\end{array}$ & 2.667 & 1.4560 & 1.3290 & 1.2270 & 1.2010 & 1.0910 & 1.0740 & 1.0570 \\
\hline & Reddy [37] & 2.639 & 1.4440 & 1.3200 & 1.2170 & 1.1900 & 1.0800 & 1.0630 & 1.0450 \\
\hline & $\begin{array}{l}\text { HSDT based IGA } \\
{[15]}\end{array}$ & 2.6297 & 1.4386 & 1.3143 & 1.2123 & 1.1855 & 1.0762 & 1.0592 & 1.0415 \\
\hline & FSDT based IGA & 2.6393 & 1.4448 & 1.3203 & 1.2176 & 1.1905 & 1.0801 & 1.0631 & 1.0452 \\
\hline & $\begin{array}{l}\text { S-FSDT based } \\
\text { IGA }\end{array}$ & 2.6353 & 1.4428 & 1.3186 & 1.2159 & 1.1889 & 1.0785 & 1.0615 & 1.0436 \\
\hline \multirow{5}{*}{0.2} & $\begin{array}{l}\text { Elasticity } \\
\text { solutions [36] }\end{array}$ & 3.093 & 1.6580 & 1.5110 & 1.4020 & 1.3750 & 1.2620 & 1.2440 & 1.2260 \\
\hline & Reddy [37] & 2.979 & 1.6130 & 1.4730 & 1.3620 & 1.3330 & 1.2160 & 1.1990 & 1.1800 \\
\hline & $\begin{array}{l}\text { HSDT based IGA } \\
{[15]}\end{array}$ & 2.9541 & 1.5958 & 1.4557 & 1.3467 & 1.3187 & 1.2060 & 1.1884 & 1.1700 \\
\hline & FSDT based IGA & 2.9799 & 1.6137 & 1.4738 & 1.3622 & 1.3332 & 1.2166 & 1.1988 & 1.1800 \\
\hline & $\begin{array}{l}\text { S-FSDT based } \\
\text { IGA }\end{array}$ & 2.9625 & 1.6051 & 1.4659 & 1.3548 & 1.3260 & 1.2097 & 1.1918 & 1.1732 \\
\hline
\end{tabular}

Table 9. First normalized natural frequency of $\mathrm{Al} / \mathrm{Al}_{2} \mathrm{O}_{3}$ square plate for $a / h=5$.

\begin{tabular}{|c|c|c|c|c|c|}
\hline & Method & Number of control points & $n=1$ & $n=4$ & $n=10$ \\
\hline \multirow{5}{*}{\multicolumn{2}{|c|}{ S-FSDT based IGA }} & $6 \times 6$ & 0.1625 & 0.1391 & 0.1321 \\
\hline & & $8 \times 8$ & 0.1625 & 0.1389 & 0.1320 \\
\hline & & $12 \times 12$ & 0.1624 & 0.1390 & 0.1320 \\
\hline & & $16 \times 16$ & 0.1624 & 0.1390 & 0.1320 \\
\hline & & $24 \times 24$ & 0.1624 & 0.1390 & 0.1320 \\
\hline \multicolumn{2}{|r|}{ FSDT based IGA } & $16 \times 16$ & 0.1630 & 0.1398 & 0.1323 \\
\hline \multirow{3}{*}{ Exact } & 2D-HOT [38] & - & 0.1640 & 0.1383 & 0.1306 \\
\hline & S-HSDT [39] & - & 0.1631 & 0.1378 & 0.1301 \\
\hline & S-FSDT based FEM [40] & - & 0.1629 & 0.1396 & 0.1322 \\
\hline
\end{tabular}


Table 10. First normalized natural frequency of $\mathrm{Al} / \mathrm{Al}_{2} \mathrm{O}_{3}$ square plate for $a / h=2,10,20$.

\begin{tabular}{|c|c|c|c|c|c|c|c|}
\hline$a / h$ & \multicolumn{2}{|c|}{ Method } & $n=0$ & $n=0.5$ & $n=1$ & $n=4$ & $n=10$ \\
\hline \multirow{5}{*}{2} & \multirow{2}{*}{$\begin{array}{l}\text { Analytical } \\
\text { solutions }\end{array}$} & $\begin{array}{l}\text { 2D-HOT } \\
{[38]}\end{array}$ & 0.9400 & 0.8232 & 0.7476 & 0.5997 & 0.5460 \\
\hline & & $\begin{array}{l}\text { S-HSDT } \\
{[39]}\end{array}$ & 0.9297 & 0.8110 & 0.7356 & 0.5924 & 0.5412 \\
\hline & \multicolumn{2}{|c|}{ S-FSDT based FEM [40] } & 0.9255 & 0.8052 & 0.7330 & 0.6115 & 0.5642 \\
\hline & \multicolumn{2}{|c|}{ FSDT based IGA } & 0.9265 & 0.8060 & 0.7330 & 0.6111 & 0.5640 \\
\hline & \multicolumn{2}{|c|}{ S-FSDT based IGA } & 0.9265 & 0.8027 & 0.7267 & 0.6055 & 0.5620 \\
\hline \multirow{5}{*}{10} & \multirow{2}{*}{$\begin{array}{l}\text { Analytical } \\
\text { solutions }\end{array}$} & $\begin{array}{l}\text { 2D-HOT } \\
{[38]}\end{array}$ & 0.0578 & 0.0492 & 0.0443 & 0.0381 & 0.0364 \\
\hline & & $\begin{array}{l}\text { S-HSDT } \\
{[39]}\end{array}$ & 0.0577 & 0.0490 & 0.0442 & 0.0381 & 0.0364 \\
\hline & \multicolumn{2}{|c|}{ S-FSDT based FEM [40] } & 0.0576 & 0.0489 & 0.0441 & 0.0382 & 0.0365 \\
\hline & \multicolumn{2}{|c|}{ FSDT based IGA } & 0.0577 & 0.0490 & 0.0442 & 0.0382 & 0.0366 \\
\hline & \multicolumn{2}{|c|}{ S-FSDT based IGA } & 0.0577 & 0.0490 & 0.0441 & 0.0382 & 0.0365 \\
\hline \multirow{5}{*}{20} & \multicolumn{2}{|c|}{$\begin{array}{l}\text { 2D-HOT based analytical } \\
\text { solutions [38] }\end{array}$} & 0.0148 & 0.0125 & 0.0113 & 0.0098 & 0.0094 \\
\hline & \multicolumn{2}{|c|}{$\begin{array}{l}\text { FSDT based element-free } \\
\text { kp-Ritz method [41] }\end{array}$} & 0.0146 & 0.0124 & 0.0112 & 0.0097 & 0.0093 \\
\hline & \multicolumn{2}{|c|}{ S-FSDT based FEM [40] } & 0.0148 & 0.0125 & 0.0113 & 0.0098 & 0.0094 \\
\hline & \multicolumn{2}{|c|}{ FSDT based IGA } & 0.0148 & 0.0125 & 0.0113 & 0.00981 & 0.0094 \\
\hline & \multicolumn{2}{|c|}{ S-FSDT based IGA } & 0.0148 & 0.0125 & 0.0113 & 0.00981 & 0.0094 \\
\hline
\end{tabular}


Table 11. First five mode normalized natural frequencies of $\mathrm{Al} / \mathrm{Al}_{2} \mathrm{O}_{3}$ thin plate with various boundary conditions and gradient indexes.

(a) SFSF

\begin{tabular}{|c|c|c|c|c|c|c|}
\hline$n$ & Method & Mode 1 & Mode 2 & Mode 3 & Mode 4 & Mode 5 \\
\hline \multirow{4}{*}{0} & CPT-neu based IGA [42] & 56.5660 & 94.7565 & 215.6539 & 228.7079 & 274.4598 \\
\hline & FSDT based IGA & 56.5526 & 94.6609 & 215.3651 & 228.5948 & 274.1872 \\
\hline & S-FSDT based IGA & 56.5584 & 94.7388 & 215.5711 & 228.5829 & 274.2876 \\
\hline & Exact [43] & 56.4791 & 94.7141 & 215.6299 & - & - \\
\hline \multirow{4}{*}{0.5} & CPT-neu based IGA [42] & 47.8972 & 80.2349 & 182.6041 & 193.6578 & 232.3979 \\
\hline & FSDT based IGA & 47.8872 & 80.1627 & 182.3880 & 193.5819 & 232.2005 \\
\hline & S-FSDT based IGA & 47.8913 & 80.2210 & 182.5386 & 193.5617 & 232.2649 \\
\hline & Exact $[43]$ & 47.7452 & 80.1576 & 182.4411 & - & - \\
\hline \multirow{4}{*}{1} & CPT-neu based IGA [42] & 43.1596 & 72.2984 & 164.5401 & 174.5012 & 209.4085 \\
\hline & FSDT based IGA & 43.1511 & 72.2369 & 164.3570 & 174.4412 & 209.2445 \\
\hline & S-FSDT based IGA & 43.1544 & 72.2861 & 164.4815 & 174.4179 & 209.2924 \\
\hline & Exact $[43]$ & 43.0872 & 72.2001 & 164.3911 & - & - \\
\hline \multirow{4}{*}{2} & CPT-neu based IGA [42] & 39.2395 & 65.7314 & 149.5922 & 158.6496 & 190.3849 \\
\hline & FSDT based IGA & 39.2316 & 65.6747 & 149.4233 & 158.5933 & 190.2330 \\
\hline & S-FSDT based IGA & 39.2347 & 65.7197 & 149.5365 & 158.5722 & 190.2767 \\
\hline & Exact [43] & 39.1666 & 65.6400 & 149.0583 & - & - \\
\hline
\end{tabular}

(b) SSSS

\begin{tabular}{|c|l|c|c|c|c|c|}
\hline$n$ & \multicolumn{1}{|c|}{ Method } & Mode 1 & Mode 2 & Mode 3 & Mode 4 & Mode 5 \\
\hline \multirow{2}{*}{0} & CPT-neu based IGA [42] & 115.9253 & 289.7845 & 289.7845 & 463.5955 & 579.5359 \\
\cline { 2 - 7 } & FSDT based IGA & 115.8932 & 289.6145 & 289.6145 & 463.1163 & 579.2636 \\
\cline { 2 - 7 } & S-FSDT based IGA & 115.8926 & 289.5806 & 289.5806 & 463.0741 & 578.7215 \\
\hline
\end{tabular}




\begin{tabular}{|c|c|c|c|c|c|c|}
\hline & Exact [43] & 115.8695 & 289.7708 & - & 463.4781 & - \\
\hline \multirow{4}{*}{0.5} & CPT-neu based IGA [42] & 98.1595 & 245.3740 & 245.3740 & 392.5471 & 490.7185 \\
\hline & FSDT based IGA & 98.1354 & 245.2532 & 245.2532 & 392.195 & 490.6291 \\
\hline & S-FSDT based IGA & 98.1343 & 245.2169 & 245.2169 & 392.1448 & 490.0963 \\
\hline & Exact [43] & 98.0136 & 245.3251 & - & 392.4425 & - \\
\hline \multirow{4}{*}{1} & CPT-neu based IGA [42] & 88.4501 & 221.1011 & 221.1011 & 353.7127 & 442.1697 \\
\hline & FSDT based IGA & 88.4296 & 221.0019 & 221.0019 & 353.4173 & 442.1505 \\
\hline & S-FSDT based IGA & 88.428 & 220.9643 & 220.9643 & 353.3613 & 441.6348 \\
\hline & Exact [43] & 88.3093 & 221.0643 & - & 353.6252 & - \\
\hline \multirow{4}{*}{2} & CPT-neu based IGA [42] & 80.4160 & 201.0155 & 201.0155 & 321.5761 & 401.9929 \\
\hline & FSDT based IGA & 80.3972 & 200.9234 & 200.9234 & 321.3032 & 401.9628 \\
\hline & S-FSDT based IGA & 80.3953 & 200.8879 & 200.8879 & 321.2475 & 401.5008 \\
\hline & Exact [43] & 80.3517 & 200.8793 & - & 321.4069 & - \\
\hline
\end{tabular}

(c) SCSC

\begin{tabular}{|c|l|c|c|c|c|c|}
\hline$n$ & \multicolumn{1}{|c|}{ Method } & Mode 1 & Mode 2 & Mode 3 & Mode 4 & Mode 5 \\
\hline \multirow{2}{*}{0} & CPT-neu based IGA [42] & 170.0240 & 321.4690 & 407.1180 & 555.3781 & 600.2165 \\
\cline { 2 - 7 } & FSDT based IGA & 169.8890 & 321.1655 & 406.5798 & 554.3949 & 599.7747 \\
\cline { 2 - 7 } & S-FSDT based IGA & 169.9230 & 321.1937 & 406.5707 & 554.5021 & 599.3170 \\
\cline { 2 - 7 } & Exact [43] & 170.0196 & 321.4069 & - & 555.2809 & - \\
\hline \multirow{2}{*}{0.5} & CPT-neu based IGA [42] & 143.9675 & 272.2028 & 344.7257 & 470.2635 & 508.2296 \\
\cline { 2 - 7 } & FSDT based IGA & 143.8672 & 271.9835 & 344.3519 & 469.5518 & 508.0145 \\
\cline { 2 - 7 } & S-FSDT based IGA & 143.8904 & 271.9916 & 344.3090 & 469.5928 & 507.5430 \\
\cline { 2 - 7 } & Exact [43] & 143.8179 & 272.1090 & - & 470.0770 & - \\
\hline \multirow{2}{*}{1} & FSDT based IGA & 129.6422 & 245.0938 & 310.3216 & 423.1484 & 457.8229 \\
\cline { 2 - 7 } & S-FSDT based IGA & 129.6605 & 245.0927 & 310.2664 & 423.1599 & 457.3585 \\
\hline
\end{tabular}




\begin{tabular}{|l|l|c|c|c|c|c|}
\hline & Exact [43] & 129.6496 & 245.1310 & - & 423.6904 & - \\
\hline \multirow{2}{*}{2} & CPT-neu based IGA [42] & 117.9435 & 222.9939 & 282.4052 & 385.2402 & 416.3375 \\
\cline { 2 - 7 } & FSDT based IGA & 117.8652 & 222.8253 & 282.1232 & 384.6922 & 416.2095 \\
\cline { 2 - 7 } & S-FSDT based IGA & 117.8818 & 222.8238 & 282.0750 & 384.7018 & 415.7952 \\
\cline { 2 - 7 } & Exact [43] & 117.8104 & 222.8111 & - & 385.0672 & - \\
\hline
\end{tabular}

(d) $\mathrm{CCCC}$

\begin{tabular}{|c|l|l|c|c|c|c|}
\hline$n$ & \multicolumn{1}{|c|}{ Method } & Mode 1 & Mode 2 & Mode 3 & Mode 4 & Mode 5 \\
\hline \multirow{2}{*}{0} & CPT-neu based IGA [42] & 211.3372 & 431.0061 & 431.0061 & 635.4464 & 772.7523 \\
\cline { 2 - 7 } & FSDT based IGA & 211.1203 & 430.3532 & 430.3532 & 633.9937 & 771.9821 \\
\cline { 2 - 7 } & S-FSDT based IGA & 211.1468 & 430.3633 & 430.3633 & 634.1625 & 770.8950 \\
\hline \multirow{2}{*}{0.5} & FSDT based IGA & 178.9493 & 364.9528 & 364.9528 & 538.0607 & 654.3228 \\
\cline { 2 - 7 } & FS-neu based IGA [42] & 178.7886 & 364.4940 & 364.4940 & 537.0131 & 654.0679 \\
\cline { 2 - 7 } & S-FSDT based IGA & 178.8047 & 364.4639 & 364.4639 & 537.0816 & 652.9193 \\
\hline \multirow{2}{*}{1} & CPT-neu based IGA [42] & 161.2484 & 328.8502 & 328.8502 & 484.8293 & 589.5860 \\
\cline { 2 - 7 } & FSDT based IGA & 161.1129 & 328.4760 & 328.4760 & 483.9606 & 589.5289 \\
\cline { 2 - 7 } & S-FSDT based IGA & 161.1242 & 328.4308 & 328.4308 & 483.9866 & 588.3962 \\
\hline \multirow{2}{*}{2} & FSDT based IGA & 146.4765 & 298.6270 & 298.6270 & 439.9730 & 535.9244 \\
\cline { 2 - 7 } & S-FSDT based IGA & 146.4868 & 298.5884 & 298.5884 & 439.9988 & 534.9293 \\
\hline
\end{tabular}




$$
\therefore
$$


1

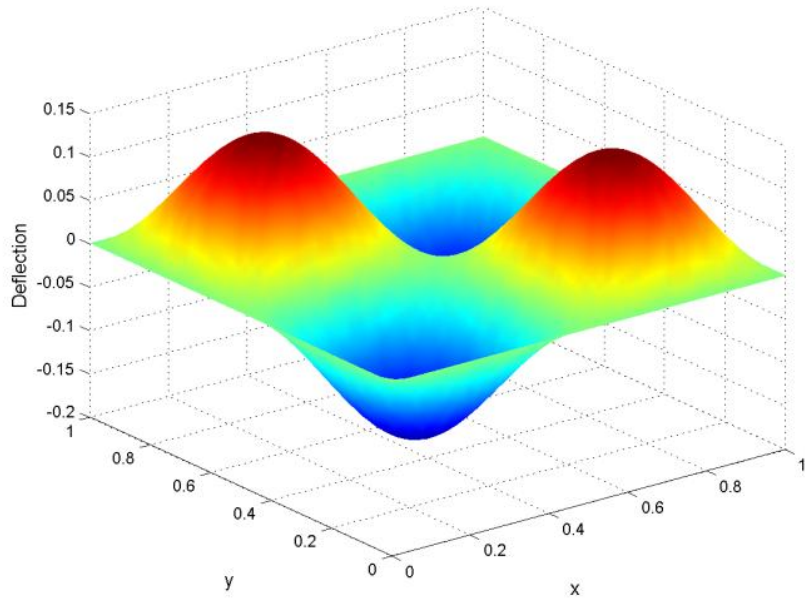

(d)

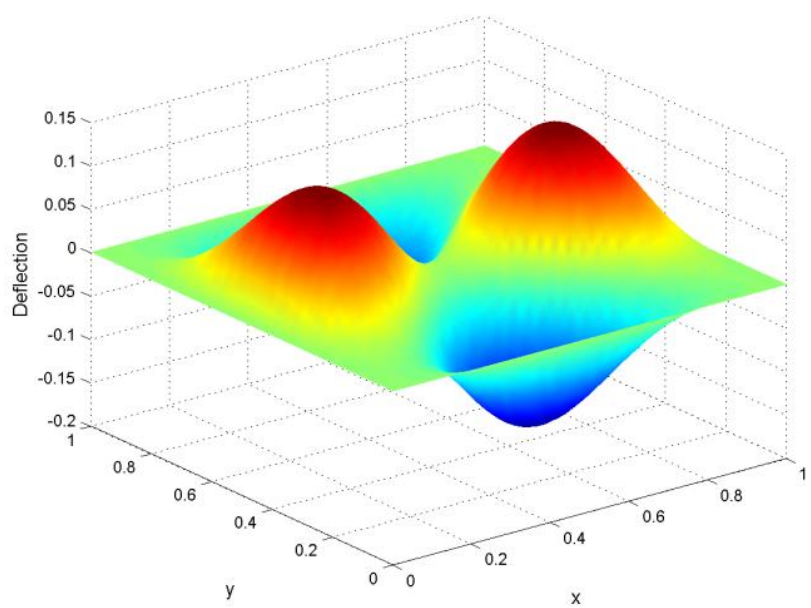

(e)

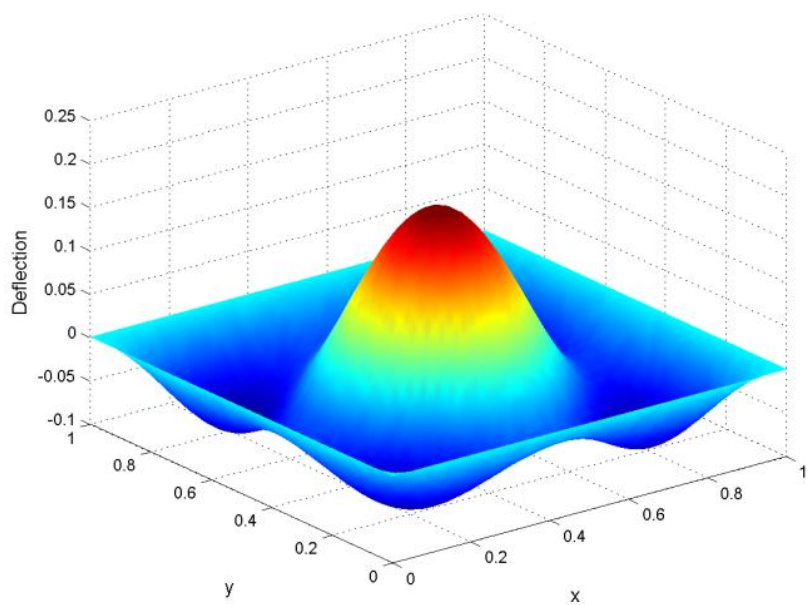

(f)

Fig. 6. First six mode shapes of simply supported $\mathrm{Al} / \mathrm{Al}_{2} \mathrm{O}_{3}$ thin plate: (a) mode 1 , (b) mode 2, (c) mode 3, (d) mode 4 , (e) mode 5, and (f) mode 6. 
1

2

3

4

5

6

7

9

10

11

12

13

14

15

16

17

18

19

20

21

22

23

24

25

26

27

28

29

30

31

32

33

34

35

36

37

38

39

40

41

42

43

44

45

46

47

48

49

50

51

52

53

54

55

56

57

58

59

60

61

62

63

64

65

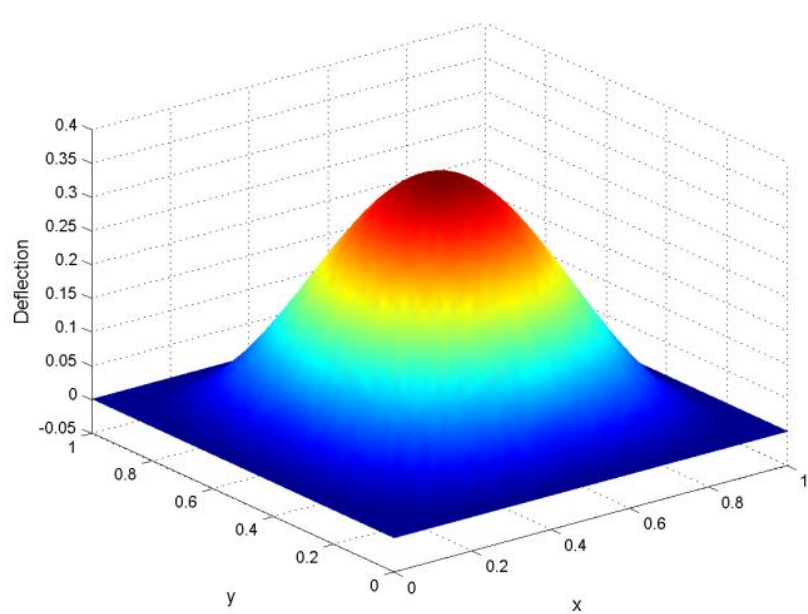

(a)

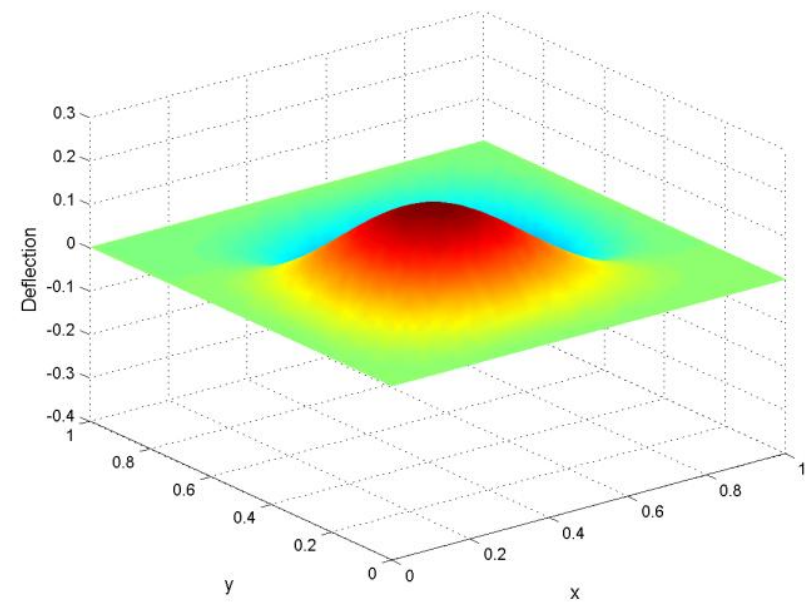

(b)

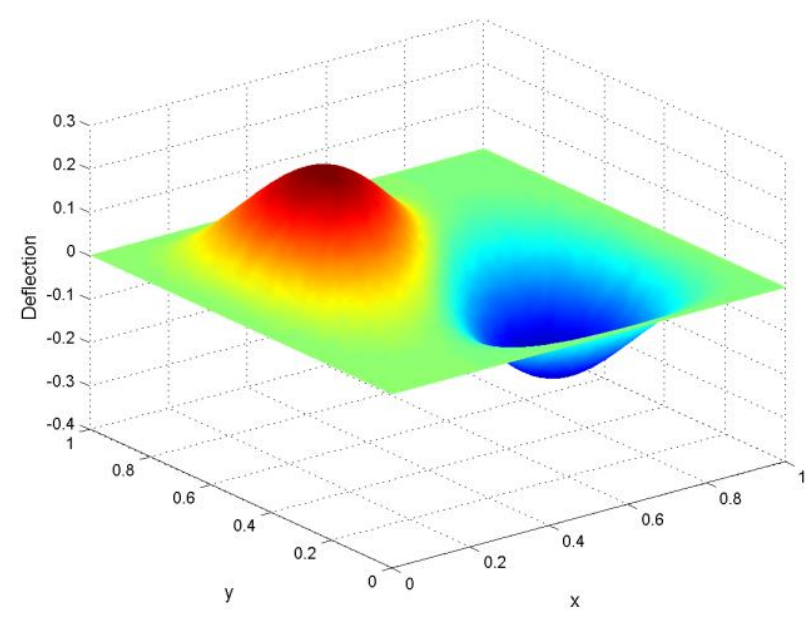

(c) 
1

2

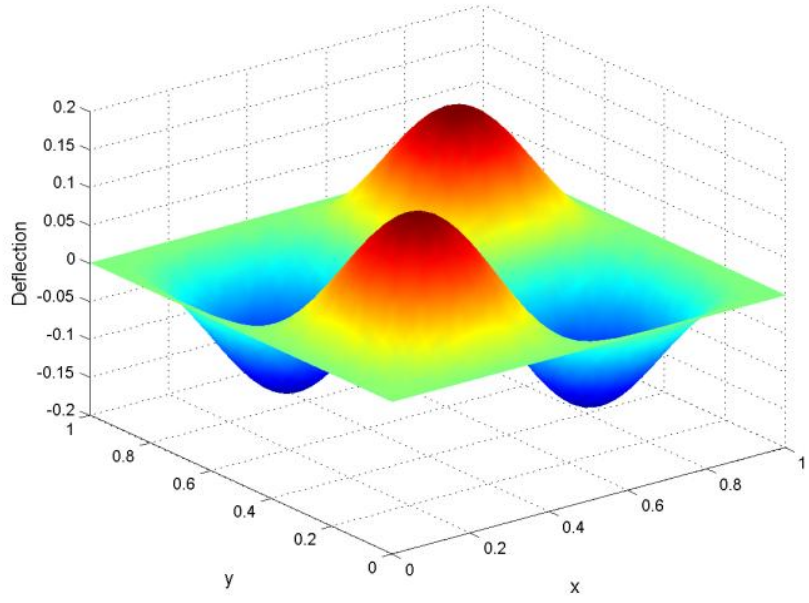

(d)

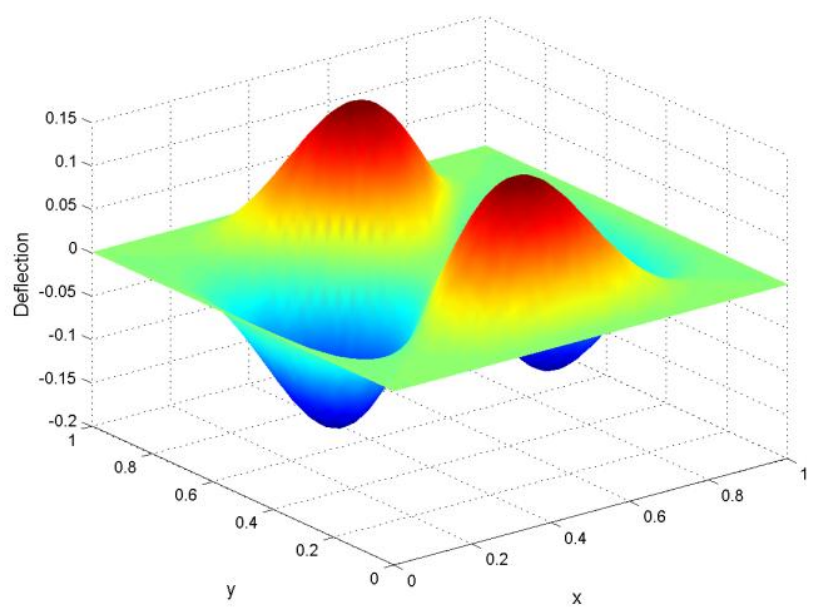

(e)

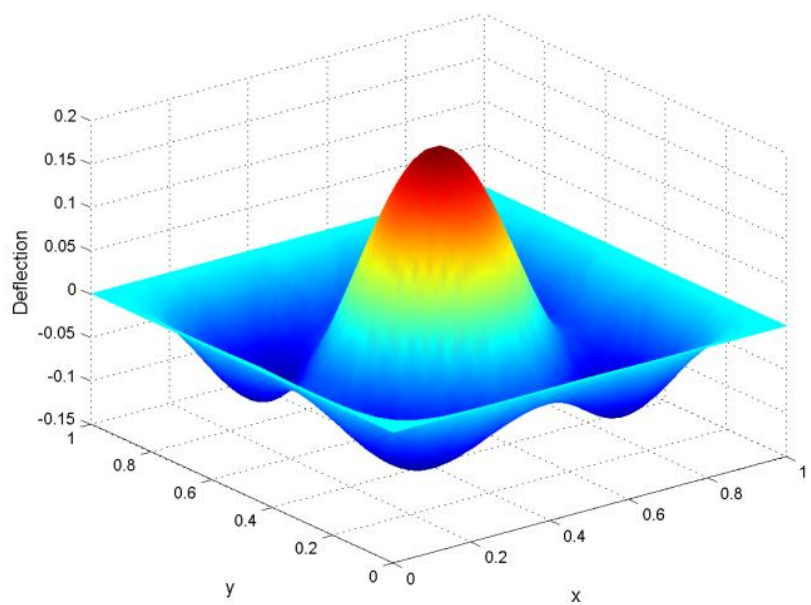

(f)

Fig. 7. First six mode shapes of clamped $\mathrm{Al} / \mathrm{Al}_{2} \mathrm{O}_{3}$ thin plate: (a) mode 1 , (b) mode 2, (c) mode 3, (d) mode 4, (e) mode 5 , and (f) mode 6. 
Table 12. First six frequencies of circular $\mathrm{Al} / \mathrm{Al}_{2} \mathrm{O}_{3}$ plate with clamped edge $(n=1)$.

\begin{tabular}{|c|c|c|c|c|c|c|c|}
\hline$h / R$ & Method & Mode 1 & Mode 2 & Mode 3 & Mode 4 & Mode 5 & Mode 6 \\
\hline \multirow{6}{*}{0.01} & semi-analytical [44] & 0.0236 & 0.0491 & 0.0805 & 0.0918 & 0.1178 & 0.1404 \\
\hline & FEM [45] & 0.0234 & 0.0486 & 0.0798 & 0.0909 & 0.1167 & 0.1391 \\
\hline & UM [45] & 0.0257 & 0.0535 & 0.0877 & 0.1000 & 0.1283 & 0.1529 \\
\hline & $\begin{array}{l}\text { HSDT based IGA } \\
{[15]}\end{array}$ & 0.0236 & 0.0492 & 0.0807 & 0.0924 & 0.1191 & 0.1431 \\
\hline & FSDT based IGA & 0.0237 & 0.0511 & 0.0855 & 0.0997 & 0.1101 & 0.1451 \\
\hline & S-FSDT based IGA & 0.0236 & 0.0491 & 0.0805 & 0.0919 & 0.1180 & 0.1408 \\
\hline \multirow{6}{*}{0.1} & semi-analytical [44] & 2.3053 & 4.6934 & 7.5146 & 8.5181 & 10.7128 & 12.6197 \\
\hline & FEM [45] & 2.2888 & 4.6661 & 7.4808 & 8.4829 & 10.6776 & 12.5877 \\
\hline & UM [45] & 2.5038 & 5.0831 & 8.1156 & 9.1931 & 11.5376 & 13.5743 \\
\hline & $\begin{array}{lll}\text { IGA with HSDT } \\
{[15]}\end{array}$ & 2.3076 & 4.7005 & 7.5318 & 8.5380 & 10.7483 & 12.6636 \\
\hline & IGA with FSDT & 2.3042 & 4.6936 & 7.5190 & 8.5472 & 10.7923 & 12.8097 \\
\hline & IGA with S-FSDT & 2.3040 & 4.7137 & 7.5773 & 8.5244 & 10.8524 & 12.7017 \\
\hline \multirow{6}{*}{0.2} & semi-analytical [44] & 8.6535 & 16.7666 & 25.6486 & 28.7574 & 34.0756 & 35.0981 \\
\hline & FEM [45] & 8.6403 & 16.7890 & 25.7661 & 28.9152 & 34.1893 & 35.3618 \\
\hline & UM [45] & 9.3162 & 17.9164 & 27.2480 & 30.4998 & - & 37.1197 \\
\hline & $\begin{array}{lll}\text { IGA with HSDT } \\
{[15]}\end{array}$ & 8.6787 & 16.8595 & 25.8479 & 29.0092 & 34.0581 & 35.4875 \\
\hline & IGA with FSDT & 8.6490 & 16.7604 & 25.6426 & 28.7732 & 34.0571 & 35.1592 \\
\hline & IGA with S-FSDT & 8.6486 & 17.0016 & 26.2512 & 28.7691 & 34.1216 & 36.1557 \\
\hline
\end{tabular}


1

2

3

4

5

6

7

8

10

11

12

13

14

15

16

17

18

19

20

21

22

23

24

25

26

27

28

29

30

31

32

33

34

35

36

37

38

39

40

41

42

43

44

45

46

47

48

49

50

51

52

53

54

55

56

57

58

59

60

61

62

63

64

65

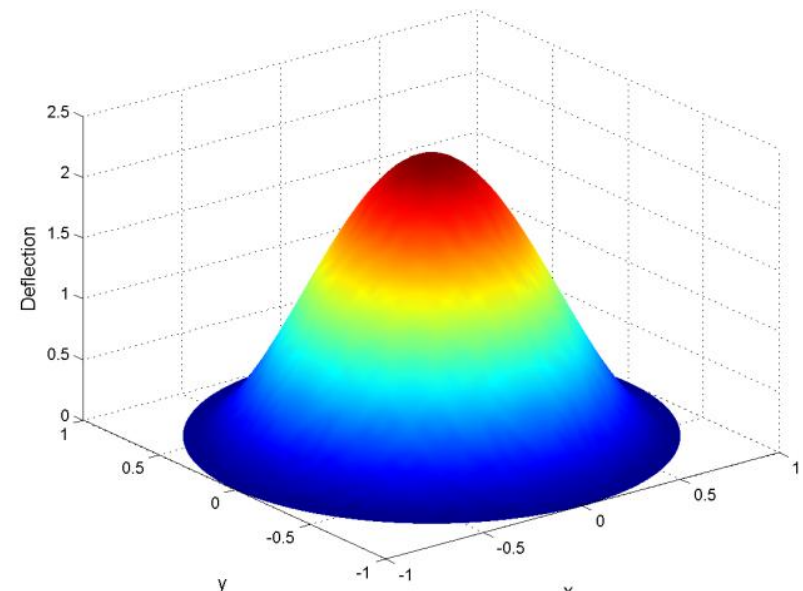

(a)

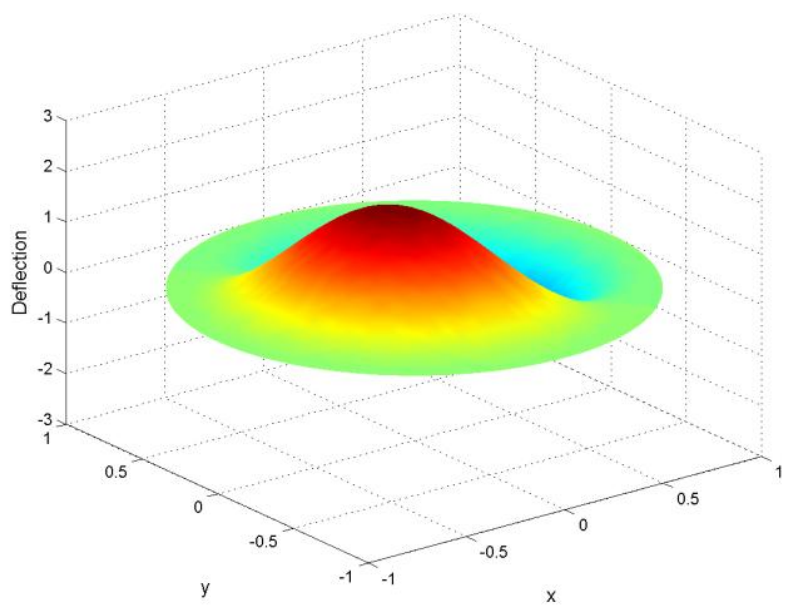

(b)

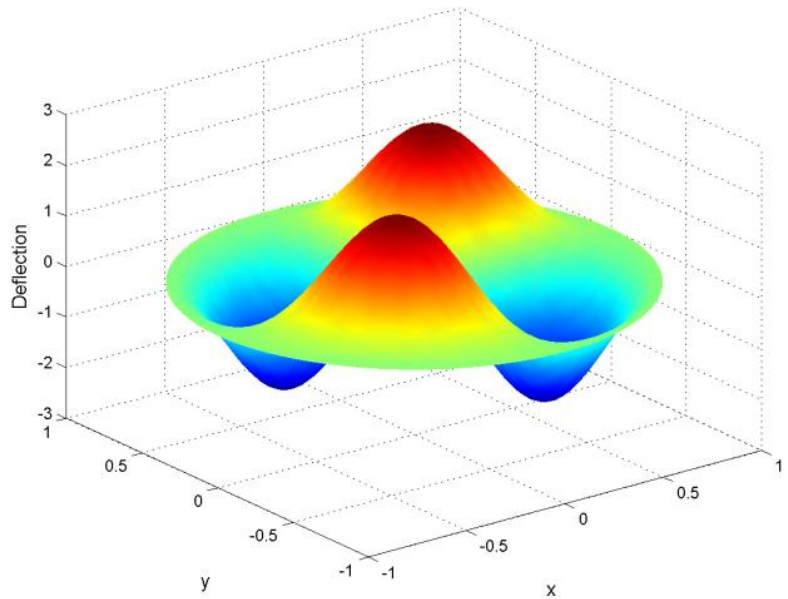

(c) 


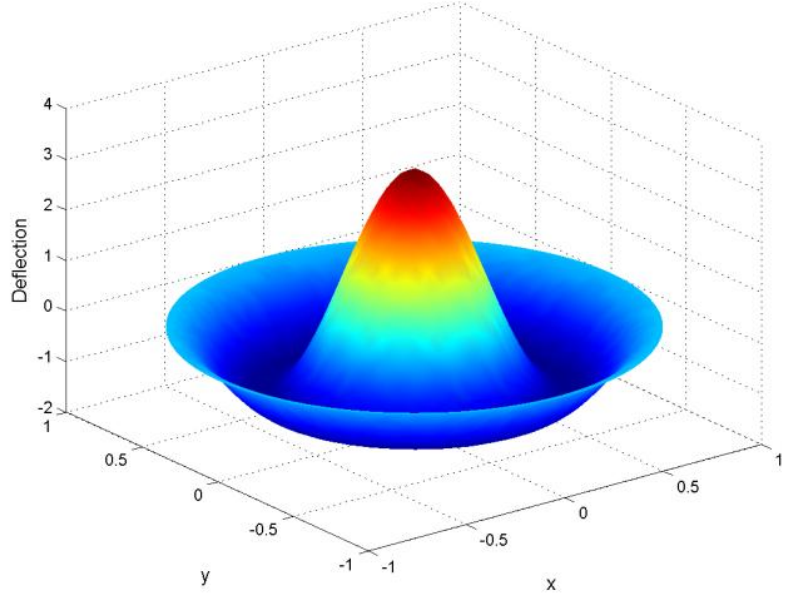

(d)

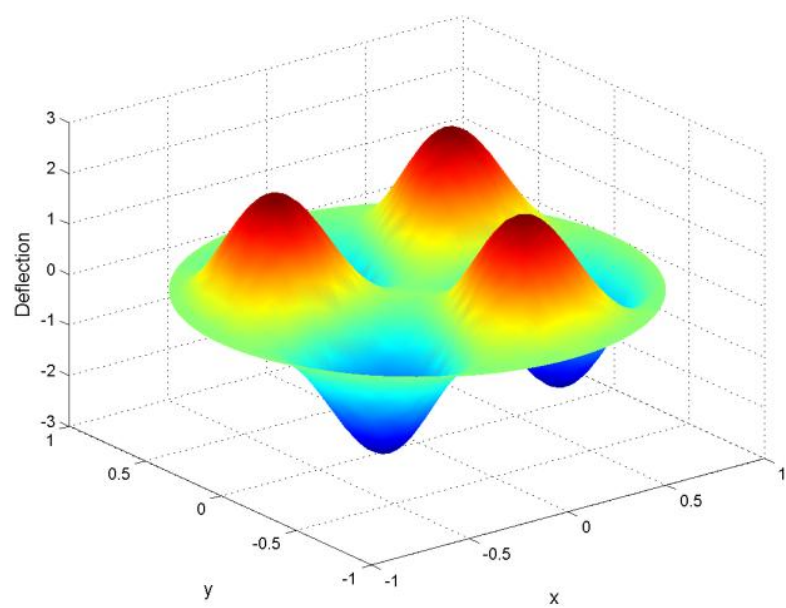

(e)

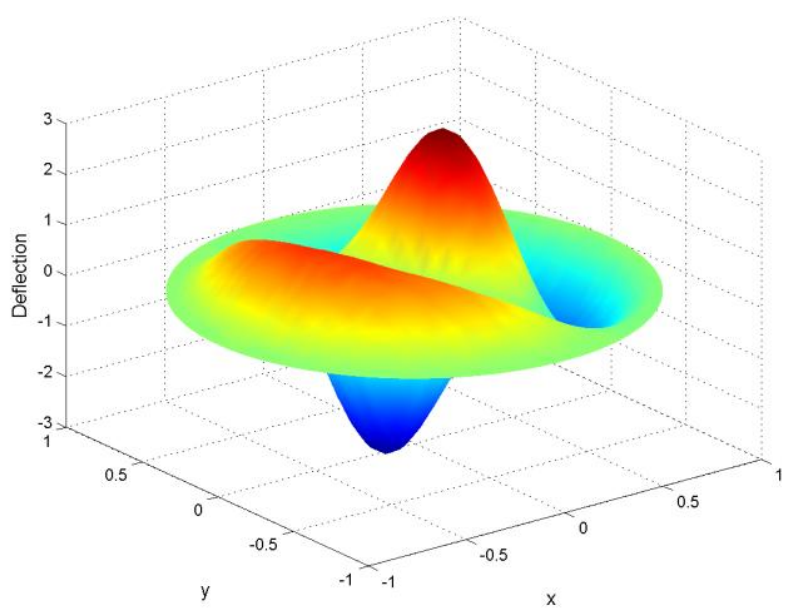

(f)

Fig. 8. First six mode shapes of clamped $\mathrm{Al} / \mathrm{Al}_{2} \mathrm{O}_{3}$ circular plate with $R / h=0.01$ and $n=1$ : (a) mode 1 , (b) mode 2 , (c) mode 3, (d) mode 4, (e) mode 5, and (f) mode 6 . 
Table 13. First normalized frequency of $\mathrm{Al} / \mathrm{Al}_{2} \mathrm{O}_{3}$ circular plate with $R / h=0.01$

\begin{tabular}{|c|l|c|c|c|c|c|}
\hline $\begin{array}{c}\text { Boundary } \\
\text { condition }\end{array}$ & \multicolumn{1}{|c|}{ Method } & $n=0$ & $n=1$ & $n=2$ & $n=5$ & $n=10$ \\
\hline \multirow{2}{*}{ Free } & FSDT based IGA & 0.01623 & 0.01239 & 0.01126 & 0.01067 & 0.01034 \\
\cline { 2 - 7 } & S-FSDT based IGA & 0.01618 & 0.01234 & 0.01122 & 0.01064 & 0.01030 \\
\hline \multirow{2}{*}{$\begin{array}{c}\text { Simply } \\
\text { supported }\end{array}$} & FSDT based IGA & 0.01495 & 0.01141 & 0.01037 & 0.00983 & 0.00952 \\
\cline { 2 - 7 } & S-FSDT based IGA & 0.01498 & 0.01214 & 0.01142 & 0.01071 & 0.01002 \\
\hline \multirow{2}{*}{ Clamped } & FSDT based IGA & 0.03106 & 0.02372 & 0.02156 & 0.02043 & 0.01977 \\
\cline { 2 - 7 } & S-FSDT based IGA & 0.03090 & 0.02358 & 0.02144 & 0.02033 & 0.01968 \\
\hline
\end{tabular}
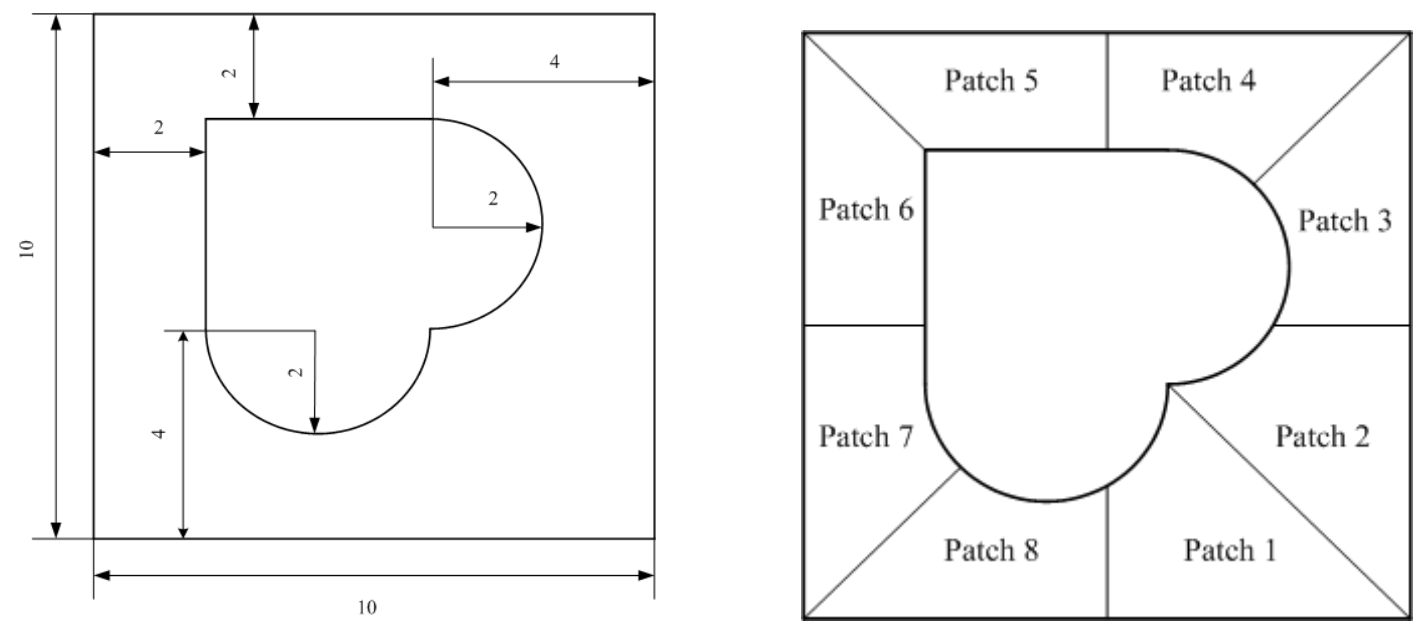

Fig. 9. A thin square plate with a complicated cutout: geometric parameters (left) and discretization of the patches (right). 

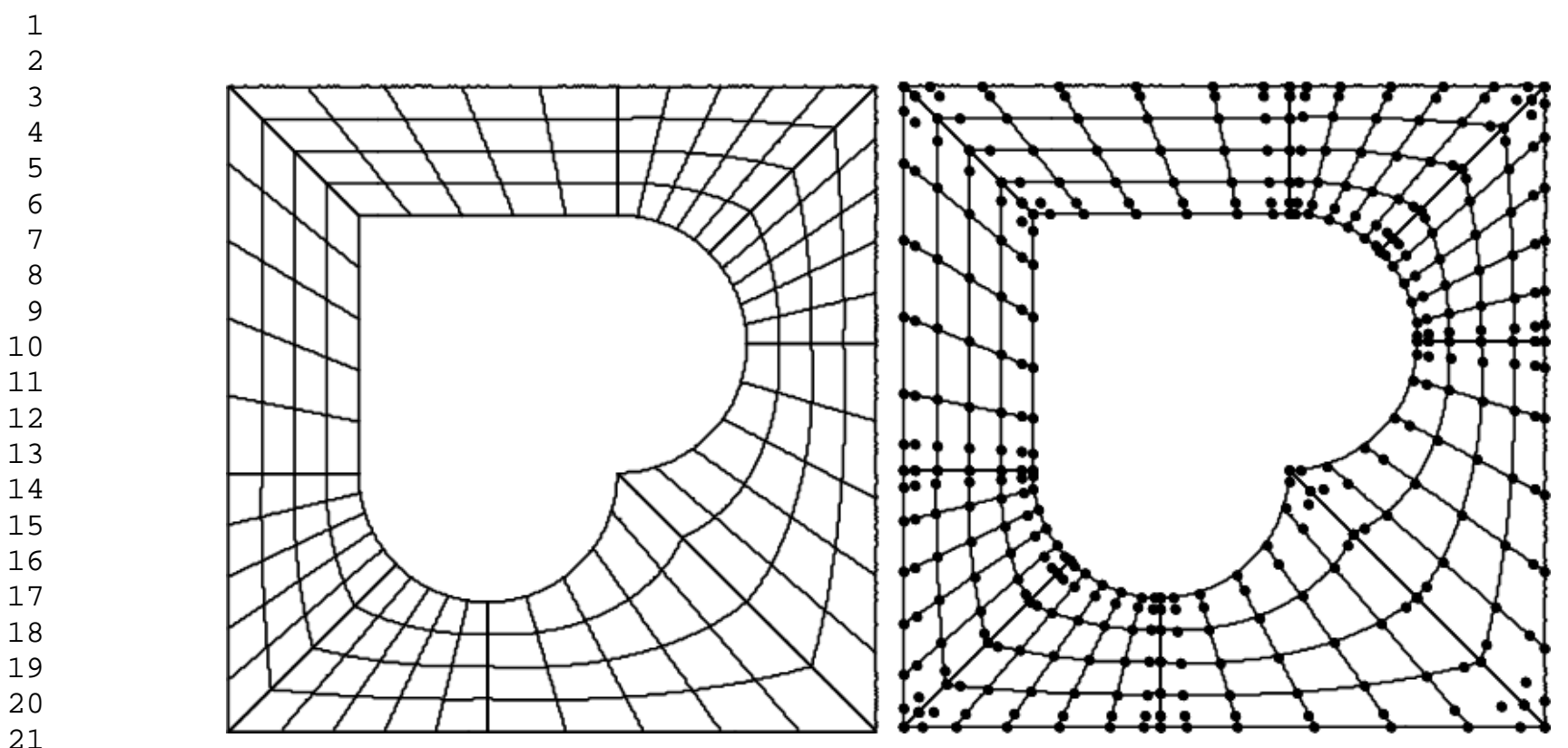

Fig. 10. Physical meshes (left) and control points (right). 
Table 14. Normalized natural frequencies of thin square plate with a complicated cutout.

(a) SSSS

\begin{tabular}{|c|c|c|c|c|c|c|}
\hline Mode & $\begin{array}{c}\text { Kirchhoff based } \\
\text { IGA [47] }\end{array}$ & MKI [48] & EFG [49] & NS-RPIM [50] & $\begin{array}{c}\text { FSDT based } \\
\text { IGA }\end{array}$ & $\begin{array}{c}\text { S-FSDT } \\
\text { based IGA }\end{array}$ \\
\hline 1 & 5.193 & 5.390 & 5.453 & 4.919 & 4.914 & 5.098 \\
\hline 2 & 6.579 & 7.502 & 8.069 & 6.398 & 6.390 & 6.608 \\
\hline 3 & 6.597 & 8.347 & 9.554 & 6.775 & 6.762 & 6.929 \\
\hline 4 & 7.819 & 10.636 & 10.099 & 8.613 & 8.568 & 8.644 \\
\hline 5 & 8.812 & 11.048 & 11.328 & 9.016 & 8.982 & 9.031 \\
\hline 6 & 9.420 & 12.894 & 12.765 & 10.738 & 10.683 & 10.591 \\
\hline 7 & 10.742 & 13.710 & 13.685 & 10.93 & 10.934 & 10.946 \\
\hline 8 & 10.776 & 14.062 & 14.305 & 11.601 & 11.694 & 11.800 \\
\hline 9 & 11.919 & 16.649 & 15.721 & 12.903 & 12.852 & 12.517 \\
\hline 10 & 13.200 & 17.364 & 17.079 & 13.283 & 13.229 & 13.001 \\
\hline
\end{tabular}

(b) $\mathrm{CCCC}$

\begin{tabular}{|c|c|c|c|c|c|}
\hline Mode & $\begin{array}{c}\text { Kirchhoff based IGA } \\
{[47]}\end{array}$ & EFG [49] & NS-RPIM [50] & FSDT based & S-FSDT based \\
\hline 1 & 7.621 & 7.548 & 7.410 & 7.453 & 7.431 \\
\hline 2 & 9.810 & 10.764 & 9.726 & 9.825 & 9.880 \\
\hline 3 & 9.948 & 11.113 & 9.764 & 9.845 & 9.992 \\
\hline 4 & 11.135 & 11.328 & 10.896 & 10.964 & 11.077 \\
\hline 5 & 11.216 & 12.862 & 11.114 & 11.165 & 11.254 \\
\hline 6 & 12.482 & 13.300 & 12.353 & 12.381 & 12.424 \\
\hline 7 & 12.872 & 14.168 & 12.781 & 12.953 & 12.862 \\
\hline 8 & 13.650 & 15.369 & 13.368 & 13.721 & 13.678 \\
\hline 9 & 14.676 & 16.205 & 14.485 & 14.511 & 14.227 \\
\hline 10 & 14.738 & 17.137 & 14.766 & 14.792 & 14.613 \\
\hline
\end{tabular}




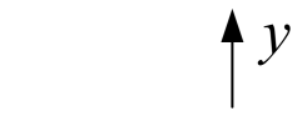

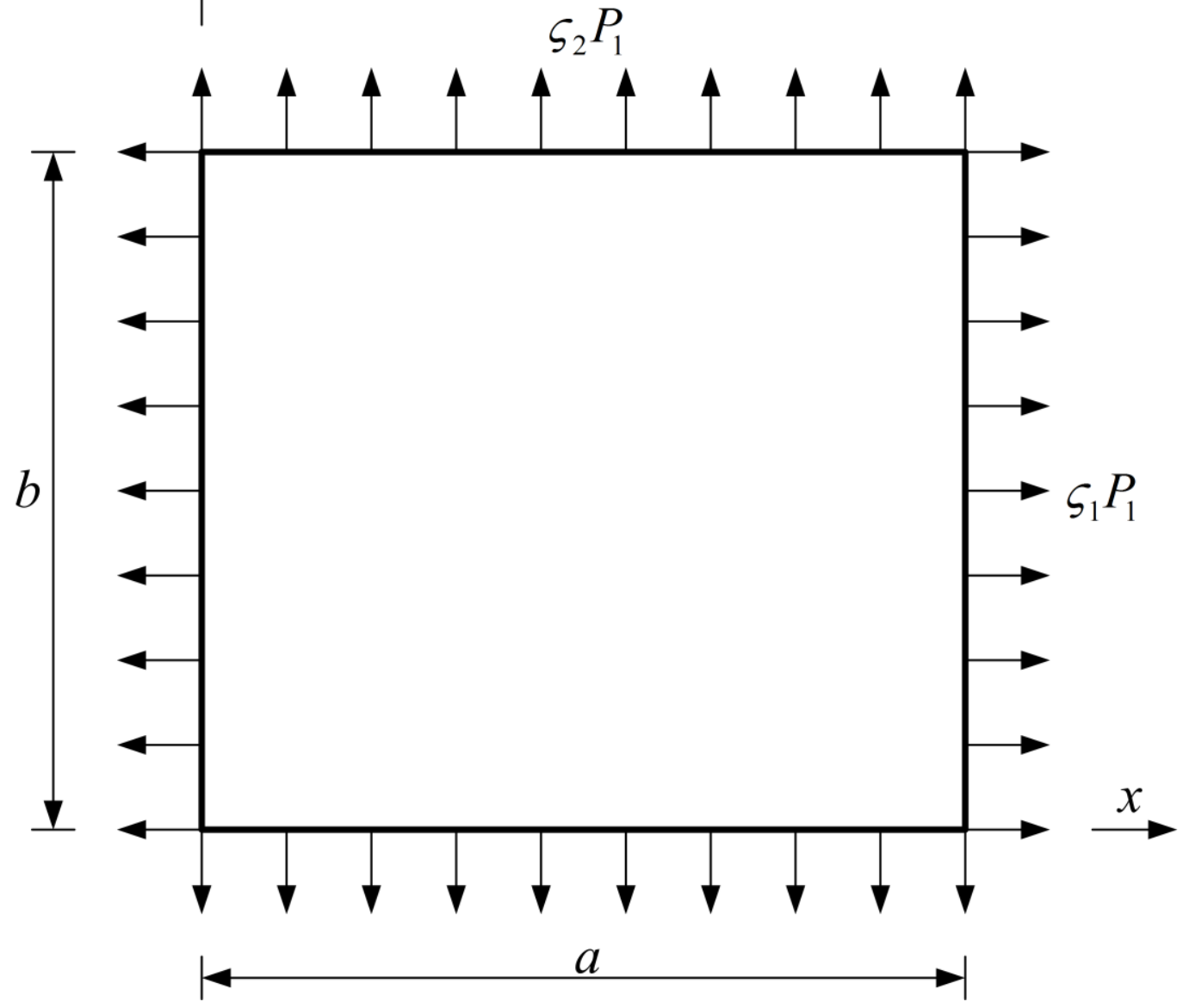

Fig. 11. A rectangular plate subjected to in-plane loads. 
Table 15. Normalized buckling loads for a homogeneous rectangular plate with different boundary conditions and aspect ratios $(b / h=0.01)$.

\begin{tabular}{|c|c|c|c|c|c|}
\hline Boundary condition & $a / b$ & $\left(\varsigma_{1}, \varsigma_{2}\right)$ & S-FSDT based IGA & Levy [51] & Levy [52] \\
\hline \multirow{4}{*}{ SSSS } & \multirow{2}{*}{0.5} & $-1,0$ & 15.4200 & 15.42 & 15.4212 \\
\hline & & $-1,-1$ & 12.3358 & 12.33 & 12.337 \\
\hline & \multirow{2}{*}{1} & $-1,0$ & 39.4720 & 39.23 & 39.4784 \\
\hline & & $-1,-1$ & 19.7360 & 19.74 & 19.7392 \\
\hline \multirow{4}{*}{ SCSC } & \multirow{2}{*}{0.5} & $-1,0$ & 18.9763 & 18.97 & 18.9775 \\
\hline & & $-1,-1$ & 14.6158 & 14.62 & 14.6174 \\
\hline & \multirow{2}{*}{1} & $-1,0$ & 75.8831 & 75.92 & 75.9099 \\
\hline & & $-1,-1$ & 37.7886 & 37.8 & 37.7996 \\
\hline \multirow{4}{*}{ SFSF } & \multirow{2}{*}{0.5} & $-1,0$ & 9.6040 & 9.604 & 9.6047 \\
\hline & & $-1,-1$ & 9.3999 & 9.4 & 9.4006 \\
\hline & \multirow{2}{*}{1} & $-1,0$ & 9.3982 & 9.4 & 9.3989 \\
\hline & & $-1,-1$ & 9.1997 & 9.199 & 9.2005 \\
\hline
\end{tabular}


Table 16. Buckling loads $(M N / m)$ for the $\mathrm{Al} / \mathrm{Al}_{2} \mathrm{O}_{3}$ plate with different boundary conditions and aspect ratios.

(a) SFSF

\begin{tabular}{|c|c|c|c|c|c|}
\hline \multirow{2}{*}{$n$} & \multirow{2}{*}{$a / b$} & \multirow{2}{*}{ Method } & \multicolumn{3}{|c|}{$\left(\varsigma_{1}, \varsigma_{2}\right)$} \\
\hline & & & 1,0 & 0,1 & 1,1 \\
\hline \multirow{6}{*}{0} & \multirow{2}{*}{0.5} & S-FSDT based IGA & 1.33648 & 3.10375 & 1.30806 \\
\hline & & Levy [52] & 1.33691 & 3.10571 & 1.30852 \\
\hline & \multirow{2}{*}{1} & S-FSDT based IGA & 0.32704 & 0.70153 & 0.32014 \\
\hline & & Levy [52] & 0.32707 & 0.701642 & 0.32016 \\
\hline & \multirow{2}{*}{1.5} & S-FSDT based IGA & 0.14316 & 0.26184 & 0.14081 \\
\hline & & Levy [52] & 0.14316 & 0.26185 & 0.15264 \\
\hline \multirow{6}{*}{1} & \multirow{2}{*}{0.5} & S-FSDT based IGA & 0.66612 & 1.54693 & 0.72216 \\
\hline & & Levy [52] & 0.66637 & 1.54801 & 0.72251 \\
\hline & \multirow{2}{*}{1} & S-FSDT based IGA & 0.16301 & 0.34967 & 0.15957 \\
\hline & & Levy [52] & 0.16302 & 0.34973 & 0.17119 \\
\hline & \multirow{2}{*}{1.5} & S-FSDT based IGA & 0.07136 & 0.13051 & 0.07019 \\
\hline & & Levy [52] & 0.07136 & 0.13052 & 0.07019 \\
\hline \multirow{6}{*}{2} & \multirow{2}{*}{0.5} & S-FSDT based IGA & 0.51977 & 1.20704 & 0.50871 \\
\hline & & Levy [52] & 0.51998 & 1.20794 & 0.50894 \\
\hline & \multirow{2}{*}{1} & S-FSDT based IGA & 0.12720 & 0.27285 & 0.12451 \\
\hline & & Levy [52] & 0.12721 & 0.27290 & 0.12452 \\
\hline & \multirow{2}{*}{1.5} & S-FSDT based IGA & 0.05568 & 0.10184 & 0.05477 \\
\hline & & Levy [52] & 0.05568 & 0.10185 & 0.05477 \\
\hline
\end{tabular}

(b) SSSS

\begin{tabular}{|c|c|c|c|c|c|}
\hline \multirow{2}{*}{$n$} & \multirow{2}{*}{$a / b$} & \multirow{2}{*}{ Method } & \multicolumn{3}{|c|}{$\left(\varsigma_{1}, \varsigma_{2}\right)$} \\
\hline & & & 1,0 & 0,1 & 1,1 \\
\hline \multirow{6}{*}{0} & \multirow{2}{*}{0.5} & S-FSDT based IGA & 2.14581 & 8.58058 & 1.71654 \\
\hline & & Levy [52] & 2.14655 & 8.58619 & 1.71724 \\
\hline & \multirow{2}{*}{1} & S-FSDT based IGA & 1.37357 & 1.37357 & 0.68678 \\
\hline & & Levy [52] & 1.37379 & 1.37379 & 0.68689 \\
\hline & \multirow{2}{*}{1.5} & S-FSDT based IGA & 1.49042 & 0.71653 & 0.49603 \\
\hline & & Levy [52] & 1.49066 & 0.71658 & 0.49609 \\
\hline \multirow{6}{*}{1} & \multirow{2}{*}{0.5} & S-FSDT based IGA & 1.06949 & 4.27663 & 0.85554 \\
\hline & & Levy [52] & 1.06993 & 4.27971 & 0.85594 \\
\hline & \multirow{2}{*}{1} & S-FSDT based IGA & 0.68463 & 0.68463 & 0.34231 \\
\hline & & Levy [52] & 0.68475 & 0.684753 & 0.34238 \\
\hline & \multirow{2}{*}{1.5} & S-FSDT based IGA & 0.74286 & 0.35714 & 0.24724 \\
\hline & & Levy [52] & 0.743 & 0.35717 & 0.24727 \\
\hline 2 & 0.5 & S-FSDT based IGA & 0.83450 & 3.33699 & 0.66756 \\
\hline
\end{tabular}


(c)SCSC

\begin{tabular}{|c|c|c|c|c|c|}
\hline \multirow{2}{*}{$n$} & \multirow{2}{*}{$a / b$} & \multirow{2}{*}{ Method } & \multicolumn{3}{|c|}{$\left(\varsigma_{1}, \varsigma_{2}\right)$} \\
\hline & & & 1,0 & 0,1 & 1,1 \\
\hline \multirow{6}{*}{0} & \multirow{2}{*}{0.5} & S-FSDT based IGA & 2.64052 & 6.65596 & 2.03369 \\
\hline & & Levy [52] & 2.64155 & 6.65897 & 2.03466 \\
\hline & \multirow{2}{*}{1} & S-FSDT based IGA & 2.64062 & 2.31569 & 1.31499 \\
\hline & & Levy [52] & 2.64155 & 2.31593 & 1.31537 \\
\hline & \multirow{2}{*}{1.5} & S-FSDT based IGA & 2.44312 & 1.72986 & 1.29362 \\
\hline & & Levy [52] & 2.44395 & 1.72987 & 1.29397 \\
\hline \multirow{6}{*}{1} & \multirow{2}{*}{0.5} & S-FSDT based IGA & 1.31606 & 3.31722 & 1.01360 \\
\hline & & Levy [52] & 1.31666 & 3.3191 & 1.01416 \\
\hline & \multirow{2}{*}{1} & S-FSDT based IGA & 1.31612 & 1.15417 & 0.65541 \\
\hline & & Levy [52] & 1.31666 & 1.15435 & 0.65563 \\
\hline & \multirow{2}{*}{1.5} & S-FSDT based IGA & 1.21771 & 0.86219 & 0.64477 \\
\hline & & Levy [52] & 1.2181 & 0.86224 & 0.64496 \\
\hline \multirow{6}{*}{2} & \multirow{2}{*}{0.5} & S-FSDT based IGA & 1.02691 & 2.58828 & 0.79089 \\
\hline & & Levy [52] & 1.02741 & 2.58995 & 0.79136 \\
\hline & \multirow{2}{*}{1} & S-FSDT based IGA & 1.02696 & 0.90059 & 0.51141 \\
\hline & & Levy [52] & 1.02741 & 0.90076 & 0.511603 \\
\hline & \multirow{2}{*}{1.5} & S-FSDT based IGA & 0.95018 & 0.67276 & 0.50311 \\
\hline & & Levy [52] & 0.95056 & 0.67282 & 0.50328 \\
\hline
\end{tabular}

